\title{
Myelin Loss and Axonal Ion Channel Adaptations Associated with Gray Matter Neuronal Hyperexcitability
}

\author{
Mustafa S. Hamada ${ }^{1}$ and $\odot$ Maarten H.P. Kole ${ }^{1,2}$ \\ ${ }^{1}$ Department of Axonal Signaling, Netherlands Institute for Neuroscience, Royal Netherlands Academy of Arts and Sciences, 1105 BA Amsterdam, The \\ Netherlands, and ${ }^{2}$ Cell Biology, Faculty of Science, Utrecht University, 3584 CH Utrecht, The Netherlands
}

Myelination and voltage-gated ion channel clustering at the nodes of Ranvier are essential for the rapid saltatory conduction of action potentials. Whether myelination influences the structural organization of the axon initial segment (AIS) and action potential initiation is poorly understood. Using the cuprizone mouse model, we combined electrophysiological recordings with immunofluorescence of the voltage-gated Nav1.6 and Kv7.3 subunits and anchoring proteins to analyze the functional and structural properties of single demyelinated neocortical L5 axons. Whole-cell recordings demonstrated that neurons with demyelinated axons were intrinsically more excitable, characterized by increased spontaneous suprathreshold depolarizations as well as antidromically propagating action potentials ectopically generated in distal parts of the axon. Immunofluorescence examination of demyelinated axons showed that $\beta I V$-spectrin, Nav1.6, and the Kv7.3 channels in nodes of Ranvier either dissolved or extended into the paranodal domains. In contrast, while the AIS in demyelinated axons started more closely to the soma, ankyrin G, $\beta$ IV-spectrin, and the ion channel expression were maintained. Structure-function analysis and computational modeling, constrained by the AIS location and realistic dendritic and axonal morphologies, confirmed that a more proximal onset of the AIS slightly reduced the efficacy of action potential generation, suggesting a compensatory role. These results suggest that oligodendroglial myelination is not only important for maximizing conduction velocity, but also for limiting hyperexcitability of pyramidal neurons.

Key words: axon; axon initial segment; demyelination; Kv7.3; Nav1.6; node of Ranvier

\section{Introduction}

The development and maintenance of voltage-gated ion channel clustering to nodes of Ranvier depend on multiple and complex interactions between neurons and glia cells, including clustering of NF186 by glia-derived extracellular matrix complexes, paranodal axoglial barriers, and stabilization of ion channel proteins by cytoskeletal scaffolds (Dzhashiashvili et al., 2007; Susuki et al., 2013). As a consequence, with the lack of oligodendroglial signals, such as in the neuroinflammatory disorder multiple sclerosis (MS) or in experimental animal models of demyelination, it is well established that nodal and juxtaparanodal voltage-gated ion channels diffuse laterally and can extend into the formerly myelinated internodal regions (Foster et al., 1980; Craner et al., 2004; Waxman et al., 2004). It is thought that these voltage-gated ion

\footnotetext{
Received Nov. 20, 2014; revised March 17, 2015; accepted April 5, 2015

Author contributions: M.H.P.K. designed research; M.S.H. performed research; M.S.H. and M.H.P.K. analyzed data; M.S.H. and M.H.P.K. wrote the paper.

This work was supported by a European Research Council Starting Grant (EU $7^{\text {th }}$ Framework Program Grant Agreement number 261114) and a National Multiple Sclerosis Society grant (RG 4924A1/1). We are furthermore indebted to Dr. P. Maña and Dr. D. Liñares in the initial conceptualization phase of these experiments. We thank Sharon de Vries for the excellent technical support and are grateful to Stefan Hallermann, Arne Battefeld, Marko Popović, and Charles Cohen for critical reading and commenting on earlier versions of the manuscript.

The authors declare no competing financial interests.

This article is freely available online through the J Neurosci Author Open Choice option.

Correspondence should be addressed to Maarten H.P. Kole, Netherlands Institute for Neuroscience, Meibergdreef 47, 1105 BA Amsterdam, The Netherlands. E-mail: m.kole@nin.knaw.nl.

DOI:10.1523/JNEUROSCI.4747-14.2015

Copyright $\odot 2015$ Hamada and Kole

This is an Open Access article distributed under the terms of the Creative Commons Attribution License Creative Commons Attribution 4.0 International, which permits unrestricted use, distribution and reproduction in any medium provided that the original work is properly attributed.
}

channel reorganizations compensate, in part, for the loss of saltatory conduction and slowing of action potential (AP) propagation in white matter axons (Bostock and Sears, 1978; Felts et al., 1997). Whether myelin loss affects the intrinsic excitability of cortical gray matter neuronal circuits, where large fractions of axons in the deeper layers are myelinated (Tomassy et al., 2014), remains poorly understood.

A major hallmark of gray matter axons is the axon initial segment (AIS), a $\sim 40$ - $\mu$ m-long excitable region responsible for the initiation of the fast sodium ion-mediated AP at the onset of axons (Rasband, 2010; Kole and Stuart, 2012). AISs share many of the same ion channel proteins and assembly mechanisms of the nodes of Ranvier, including ankyrin $\mathrm{G}$, voltage-gated Nav and $\mathrm{Kv}$ channels, and cell adhesion molecules, such as neurofascin (Rasband, 2010). In contrast to nodes, the AIS develops independently of myelinating oligodendrocytes and its length is restricted by the position of submembranous intra-axonal boundaries (Mathis et al., 2001; Dzhashiashvili et al., 2007; Ogawa and Rasband, 2008; Galiano et al., 2012). To date, experimental demyelination studies have primarily focused on white matter axonal tracts and the role of myelin in the nodal organization, but the impact on the AIS is not understood.

To test the role of myelination in the structure and function of the AIS, we used the cuprizone model for demyelination (Kipp et al., 2009) and examined the myelinated axons of thick-tufted L5 pyramidal neurons in the somatosensory cortex. Demyelination relocated the position of the AIS toward the soma and reduced Nav1.6 channels but was also associated with increased Kv7.3 expression into the internodes, and AP initiation was only slightly 
impaired. Notably, demyelination facilitated the initiation of ectopic APs antidromically propagating into the AIS and somatodendritic domain. These results suggest that oligodendroglial myelination is not only important for maximizing conduction velocity, but also for spatiotemporally confining AP generation to the AIS and limiting hyperexcitability of cortical pyramidal neurons.

\section{Materials and Methods}

Animals. Male C57BL/ 6 mice (Harlan) at 6 weeks of age ( $\sim 20 \mathrm{~g})$ were fed ad libitum 0.2 or $0.3 \%(\mathrm{w} / \mathrm{w})$ cuprizone (biscyclohexane oxaldihydrazone, Sigma-Aldrich Chemie) milled into mouse chow for 5 or 9 weeks to induce acute and chronic demyelination, respectively. The body weight loss of cuprizone-treated mice was $20 \pm 2.3 \%(n=89)$ and $25 \pm 2.0 \%$ $(n=39)$ with 0.2 and $0.3 \%$ cuprizone, respectively. Control mice received either powder or normal chow food. All experiments were done in compliance with the European Communities Council Directive of 24 November 1986 (86/609/EEC) and the Royal Netherlands Academy of Arts and Sciences animal welfare and ethical guidelines and protocols (DEC NIN 11.70).

Electrophysiological recording. At the end of the cuprizone feeding, mice were decapitated under deep isoflurane anesthesia (3\%) and the brain was quickly removed from the skull and placed into ice-cold slicing artificial CSF (aCSF) of the following composition (in mM): $125 \mathrm{NaCl}, 3$ $\mathrm{KCl}, 25$ glucose, $25 \mathrm{NaHCO}_{3}, 1.25 \mathrm{Na}_{2} \mathrm{H}_{2} \mathrm{PO}_{4}, 1 \mathrm{CaCl}_{2}, 6 \mathrm{MgCl}_{2}, 1$ kynurenic acid, saturated with $95 \% \mathrm{O}_{2}$ and $5 \% \mathrm{CO}_{2}$, $\mathrm{pH}$ 7.4. Once both hemispheres were in aCSF, the bottom one-third of the beaker was placed for a few seconds in liquid nitrogen to accelerate the cooling process. Parasagittal sections $(300 \mu \mathrm{m})$ containing the primary somatosensory cortex were cut with a Vibratome (1200S, Leica Microsystems) and brain slices were allowed a recovery period at $35^{\circ} \mathrm{C}$ for $35 \mathrm{~min}$, thereafter stored at room temperature.

Slices were transferred to an upright microscope (BX51WI, Olympus Nederland) equipped with oblique illumination optics (WI-OBCD; numerical aperture, 0.8 ). Neurons were visualized using either $40 \times$ $(0.80 \mathrm{~W})$ or $60 \times(1.00 \mathrm{~W})$ water-immersion objectives (Olympus). The microscope bath was perfused with oxygenated $\left(95 \% \mathrm{O}_{2}, 5 \% \mathrm{CO}_{2}\right)$ aCSF consisting of the following (in mM): $125 \mathrm{NaCl}, 3 \mathrm{KCl}, 25$ glucose, 25 $\mathrm{NaHCO}_{3}, 1.25 \mathrm{Na}_{2} \mathrm{H}_{2} \mathrm{PO}_{4}, 2 \mathrm{CaCl}_{2}$, and $1 \mathrm{MgCl}_{2}$. Whole-cell currentclamp and extracellular recordings were made using identical Dagan BVC-700A amplifiers (Dagan) and patch pipettes pulled from borosilicate glass (Harvard Apparatus) pulled to an open tip resistance of 5-6 $\mathrm{M} \Omega$ (whole cell) and $10 \mathrm{M} \Omega$ (extracellular recording). Patch pipettes were filled with (in $\mathrm{mm}$ ) the following: $130 \mathrm{~K}$-gluconate, $10 \mathrm{KCl}, 4 \mathrm{Mg}$ ATP, $0.3 \mathrm{Na}_{2}$-GTP, 10 HEPES, and $10 \mathrm{Na}_{2}$-phosphocreatine, $\mathrm{pH}$ 7.4, adjusted with $\mathrm{KOH}, 280 \mathrm{mOsmol} \mathrm{kg}{ }^{-1}$, to which $5 \mathrm{mg} \mathrm{ml}^{-1}$ biocytin and/or $100 \mu \mathrm{m}$ Alexa Fluor salts (488 or 594) were added.

For voltage-clamp recordings of NMDA currents, patch pipettes were filled with (in $\mathrm{mm}$ ) the following: 5 QX-314 chloride, 115 cesium methanesulfonate, 20 CsCl, 10 HEPES, 2.5 MgCl2, $4 \mathrm{Na} 2 \mathrm{ATP}$, 0.4 Na3GTP, 10 sodium phosphocreatine, and 0.6 mM EGTA, pH 7.22, $293 \mathrm{mOsmol}$ $\mathrm{kg}^{-1}$. Whole-cell voltage-clamp recordings were made using Axopatch 200B (Molecular Devices). NMDA currents were evoked by lateral extracellular stimulation using an ISO-Flex stimulus isolator (A.M.P.I.) at a distance of $200-250 \mu \mathrm{m}$ from the soma. Stimulation pipettes had open tip resistance of $5 \mathrm{M} \Omega$ and were filled with normal aCSF. All recordings were made at $34^{\circ} \mathrm{C}$.

Voltage was analog low-pass filtered at $10 \mathrm{kHz}$ (Bessel) and digitally sampled at $50-100 \mathrm{kHz}$ using an analog-to-digital converter (ITC-18, HEKA Elektronik) and data acquisition software Axograph X (v.1.5.4, Axograph Scientific). Access resistance was typically $<20 \mathrm{M} \Omega(15.1 \pm$ $0.45 \mathrm{M} \Omega, n=99)$ and fully compensated for bridge balance and capacitance. Extracellular recordings from visually identified axons were made in current-clamp mode with $0 \mathrm{pA}$ holding current and the output voltage externally amplified 50-fold. Comparisons between extracellular and intracellular recordings were corrected for the additional time lag $(60 \mu \mathrm{s})$. To visualize single axons for extracellular recordings, the epifluorescence signals were obtained with an excitation bandpass filter of 460-490 nm
(Alexa 488; emission, $520 \mathrm{~nm}$ long-pass filter) or 510-550 nm (Alexa 594; emission, $590 \mathrm{~nm}$ long-pass filter), and collected concurrently with the bright-field image. Images were acquired and automatically overlaid with a CCD camera (CoolSNAP EZ, Photometrics) controlled by an Arduino board (http://www.arduino.cc/) and $\mu$ Manager software (http://www.micro-manager.org). Approximately 100-150 sweeps were off-line aligned at the somatic AP threshold and averaged. Average spike-triggered voltage transients were aligned to the peak $\mathrm{d} V_{\mathrm{M}} / \mathrm{d} t$ of the somatic AP and axosomatic delays calculated at $20 \%$ of the peak of each averaged axonal extracellular AP, relative to that of the peak in the somatic $\mathrm{d} V_{\mathrm{M}} / \mathrm{d} t$. A $20 \%$ rise point was chosen because it is dominated by the onset of local sodium current in the AIS and nodes and not contaminated by other ionic currents, enabling reliable detection of the site of spike initiation (Palmer et al., 2010).

For analysis of spontaneous postsynaptic potentials (PSPs), individual recordings were first filtered with a high-pass filter $(0.2 \mathrm{~Hz})$, smoothed ( 10 points averaged with a moving average algorithm, Axograph $\mathrm{X}$ ), and inspected for an ideal PSP template. The template was subsequently fitted with the function $f(t)=\left[1-\exp \left(-t / \tau_{\text {rise }}\right)\right] \times \exp \left(-t / \tau_{\text {decay }}\right)$, where $\tau_{\text {rise }}$ and $\tau_{\text {decay }}$ are the rise and decay time constants. By setting a high amplitude detection threshold, the template was reiteratively applied to detect large events, which were then aligned and averaged to create the final template. The final template was then used to sweep and extract all events using a threshold-crossing protocol $\left(\sim 5-6 \times \sigma_{\text {noise }}\right)$ to automatically detect events.

Immunohistochemistry. L5 pyramidal neurons were filled with Alexa 488 or 594 and $5 \mathrm{mg} \mathrm{ml}^{-1}$ biocytin during whole-cell configuration and fixed in $-20^{\circ} \mathrm{C}$ methanol for $10 \mathrm{~min}$ (for Nav1.6, $\beta \mathrm{IV}$-spectrin, and Kv7.3 immunolabeling) or $4 \%$ paraformaldehyde (PFA) for $12 \mathrm{~h}$ at $4^{\circ} \mathrm{C}$ [for myelin basic protein (MBP)] and stored in 0.1 M PBS, pH 7.4. Sections were blocked in 5\% normal goat serum (NGS) followed by $24 \mathrm{~h}$ incubation in primary antibodies diluted in $0.1 \mathrm{~m}$ PBS containing $5 \%$ NGS and $2 \%$ Triton X-100. The following primary antibodies were used: rabbit anti-Nav1.6 and rabbit anti- $\beta$ IV-spectrin (1:100; 1:200; gift from M.N. Rasband, Baylor College of Medicine), guinea pig anti-Kv7.3 (1: 250; gift from E.C. Cooper, Baylor College of Medicine), and mouse anti-MBP (1:250; Covance). For triple immunolabeling of rabbit antiankyrin G (1:100; kind gift from M.N. Rasband, Baylor College of Medicine), mouse anti- $\alpha$-PanNav (1:100; Sigma-Aldrich Chemie), and guinea pig anti-NeuN (1:1000; EMD Millipore), 300- $\mu$ m-thick brain slices were fixed with $1 \%$ PFA for $30 \mathrm{~min}$, then blocked with $10 \%$ NGS plus $0.2 \%$ Triton X-100 diluted in $0.1 \mathrm{M}$ PBS for $2 \mathrm{~h}$ followed by $24 \mathrm{~h}$ incubation of primary antibodies, $10 \% \mathrm{NGS}$, and $0.2 \%$ Triton X-100 diluted in $0.1 \mathrm{~m}$ PBS.

For immunolabeling of Nav1.2 (1:100; Alomone Labs, catalog \#ASC002; 1:100; NeuroMab, catalog \#73-024) antibodies, mice were perfused with $0.5 \%$ PFA and $0.5 \%$ sucrose (w/v) in $0.1 \mathrm{~m}$ PBS (Tian et al., 2014). After perfusion, the brain was postfixed and subsequently immersed in $30 \%$ sucrose in 0.1 м PBS overnight. Parasagittal sections ( $40 \mu \mathrm{m}$ thick) were obtained using freezing microtome. In some experiments, acute brain slices prepared for electrophysiological recordings ( $300 \mu \mathrm{m}$ thick) were fixed with similar PFA/sucrose concentrations followed by antigen retrieval step with $0.2 \mathrm{mg} / \mathrm{ml}$ pepsin in $0.2 \mathrm{HCl}$ at $37^{\circ} \mathrm{C}$ for $15 \mathrm{~min}$ before washing steps in $0.1 \mathrm{M}$ PBS and primary antibody incubation. Furthermore, in addition to PFA fixation, for Nav1.2 immunolabeling additional acute brain slices were fixed with subzero $\left(-20^{\circ} \mathrm{C}\right)$ methanol for $10 \mathrm{~min}$ and processed as above described for Nav1.6, $\beta \mathrm{IV}$-spectrin, and Kv7.3.

For both methanol and PFA fixation methods, slices were washed in $0.1 \mathrm{M}$ PBS and incubated in the following secondary antibodies to visualize the immunoreactions: Alexa 488-conjugated goat anti-rabbit (1: 500; Invitrogen), Alexa 555 goat anti-mouse (1:500; Invitrogen), Alexa 633 goat anti-guinea pig (1:500; Invitrogen), and streptavidin Alexa 594 (1:500; Invitrogen). Sections were then mounted on glass slides and coverslipped in Vectashield (Vector Laboratories).

Images (bit depth, 8) were collected with a Leica TCS SP5 II (DMI6000 CFS; acquisition software, Leica Application Suite AF v2.6.3.8173) or SP8 X (DM6000 CFS; acquisition software, Leica Application Suite AF v3.2.1.9702) confocal laser-scanning microscopes (Leica Microsystems). Confocal images were acquired using either $40 \times$ (numerical aperture, 
A

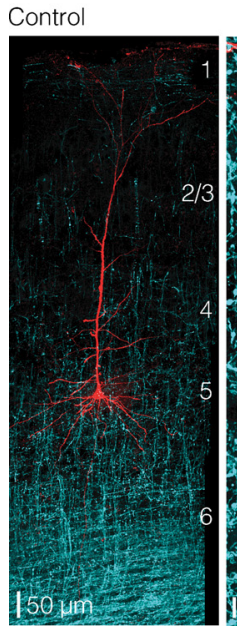

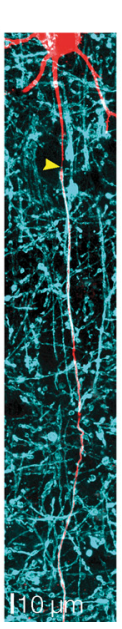
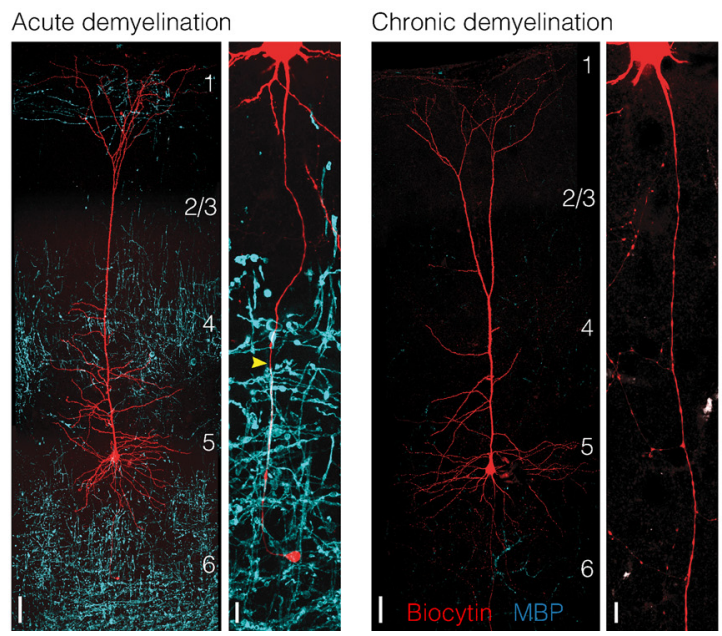

B
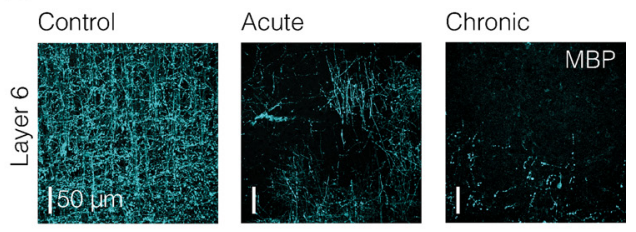

C

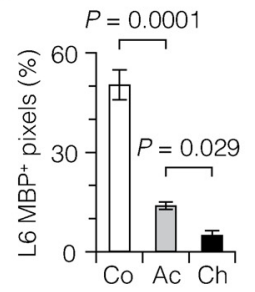

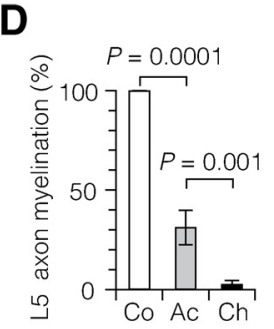

Figure 1. Graded demyelination of $L 5$ axons in the somatosensory cortex. A, Left, $z$-projected overview images of the morphology of thick-tufted $L 5$ neurons in the somatosensory cortex (biocytin fill, red) overlaid with immunofluorescence staining for MBP (cyan). Right, Higher-magnification z-projected images show the progressive demyelination of primary L5 axons. Yellow arrowheads indicate the onset of myelin. $B$, Confocal images of $L 6$ region immunolabeled for MBP expression showing the progressive loss of myelin with increasing cuprizone dosage and treatment duration. C, Quantification of MBP immunofluorescence in L6 region. Control, $n=10$; acute, $n=18$; chronic, $n=10$. D, Bar plot of the extent of myelination of single L5 axons. Control, $n=10$; acute, $n=$ 14 ; chronic, $n=16$. One-way ANOVA followed by Bonferroni's post hoc test. Co, Control; Ac, acute; Ch, chronic. Data are presented as mean \pm SEM..

1.3) or $63 \times$ (numerical aperture, 1.4 ) oil-immersion objectives. To avoid bleed-through between emission wavelengths, automated sequential acquisition of multiple channels was used. Low-magnification (objective, $40 \times$; digital zoom, $1 \times$ ) images were collected: $10-25$ images at $1 \mu \mathrm{m} z$ steps and high-magnification (objective, $60 \times$; digital zoom, $2-4 \times$ ) images were collected with $0.5-1.0 \mu \mathrm{m} z$ steps. Background noise was subtracted from each optical section before the analysis of individual AISs. The onset of the AIS was defined as the distance between the marker onset and to the edge of either the soma or a basal dendritic branch [somatic AIS, $n=74$; (basal) dendritic AIS, $n=16$ ]. The length of the AIS fluorescence profile was measured by drawing a line from the AIS onset to the distal position. All image analysis was performed using Fiji (ImageJ) graphic software (v1.47p, National Institutes of Health).

Compartmental modeling. For conductance-based multicompartmental simulations, we used a biocytin-filled thick-tufted mouse L5 pyramidal neuron from the control group (cell number 20140421_c1, 24 weeks of age), scanned at $2048 \times 2048$ pixel resolution with confocal microscopy using 1 $\mu \mathrm{m} z$ steps (Leica SP8), three-dimensionally reconstructed with Neurolucida (v.10, MicroBrightField) and imported into the Neuron simulation environment (v. 7.3; Hines and Carnevale, 2001). Both the AIS and nodal domains were carefully incorporated into the model as distinct sections with the width, and length values based on the precise location of $\beta I V$-spectrin (see Fig. $3 A-C$ ). Cytoplasmic resistance $\left(R_{\mathrm{i}}\right)$ was set to $140 \Omega \mathrm{cm}$ throughout all compartments. The resting membrane potential (RMP) was adjusted with e_pas to $-78 \mathrm{mV}$ and temperature was set nominally at $33^{\circ} \mathrm{C}$. Throughout the somatodendritic and nodal compartments, the membrane resistance $\left(R_{\mathrm{m}}\right)$ was set to $25 \mathrm{k} \Omega \mathrm{cm}^{2}$ and capacitance $\left(C_{\mathrm{m}}\right) 1.0 \mu \mathrm{F} \mathrm{cm}{ }^{-2}$. Myelination of internodal sections was represented by increasing internodal $R_{\mathrm{m}}$ to 100 $\mathrm{k} \Omega \mathrm{cm}^{2}$ and decreasing $C_{\mathrm{m}}$ to $0.25 \mu \mathrm{F} \mathrm{cm}^{-2}$, leading to a conduction velocity in the primary axons of $1.2 \mathrm{~m} \mathrm{~s}^{-1}$ in accordance to the experimental value in control $\left(\sim 1.1 \mathrm{~m} \mathrm{~s}^{-1}\right.$; see Fig. $\left.6 E\right)$. "Demyelination" was simulated by setting $R_{\mathrm{m}}$ to $25 \mathrm{k} \Omega \mathrm{cm}^{2}$ and $C_{\mathrm{m}}$ to $1.0 \mu \mathrm{F} \mathrm{cm}{ }^{-2}$ in the internodal sections, leading to a continuous axonal conduction velocity of $0.32 \mathrm{~m} \mathrm{~s}^{-1}$, consistent with experimental observations $\left(\sim 0.35 \mathrm{~m} \mathrm{~s}^{-1}\right.$; see Fig. $\left.6 E\right)$. Nav conductance was implemented by two separate eight-state allosteric models developed for the soma and the axon (Schmidt-Hieber and Bischofberger, 2010) and distributed linearly decreasing in density along the somatodendritic axis and locally increasing in peak densities in the AIS as described previously (Hallermann et al., 2012; Battefeld et al., 2014). Nav peak conductance values used were $500 \mathrm{pS} \mu \mathrm{m}^{-2}$ in the soma, $45 \mathrm{pS} \mu \mathrm{m}^{-2}$ in dendrites, $25 \mathrm{pS} \mu \mathrm{m}^{-2}$ in the internodal membrane, $8000 \mathrm{pS} \mu \mathrm{m}^{-2}$ in the nodes, and $9000 \mathrm{pS}$ $\mu \mathrm{m}^{-2}$ at the peak density in the AIS. Potassium $\left(\mathrm{K}^{+}\right)$and calcium- dependent $\mathrm{K}^{+}$conductances were distributed as described recently (Battefeld et al., 2014; see also http://senselab.med.yale.edu/modeldb/ ShowModel.asp?model=144526). Kv7 peak conductance was set at 150 pS $\mu \mathrm{m}^{-2}$ in nodes of Ranvier and at the end of the AIS, $15 \mathrm{pS} \mu \mathrm{m}^{-2}$ in axon collaterals and in the internodes, and $12 \mathrm{pS} \mu \mathrm{m}^{-2}$ in dendrites. The $\mathrm{K}^{+}$and $\mathrm{Na}^{+}$equilibrium potentials were set to -85 and $+55 \mathrm{mV}$, respectively. The hyperpolarization-activated cation channel model was exponentially increasing in the apical dendrites (Kole et al., 2006) but uniform in the axon (4 pS $\mu \mathrm{m}^{-2}$ ). The final input resistance of the model neuron was $25.4 \mathrm{M} \Omega(26.9 \mathrm{M} \Omega$, experimentally). Since we were primarily interested in the rising phase of the AP, simulations were performed with a short time step of $5 \mu \mathrm{s}$. When overlaying and comparing with experimental recordings, we applied a $10 \mathrm{kHz}$ Gaussian filter. In the experiments, APs were evoked with a 6 $\mathrm{ms}, 0.99 \mathrm{nA}$ current injection (0.94 nA in the simulations).

Statistics. Statistical significance between multiple groups was tested with GraphPad Prism (v6.0d, GraphPad Software) and Matlab R2012b (v8.0.0.783, MathWorks). The following nonparametric statistical tests were used: Mann-Whitney, Wilcoxon, Kruskal-Wallis, and Friedman tests for pharmacological experiments and immunofluorescence analysis. All other data were analyzed using parametric statistical methods. Correlation analysis was performed using IBM SPSS (v22, IBM). The cutoff significance level $(p)$ was 0.05 .

\section{Results}

Cuprizone induces dose-dependent and time-dependent gray matter demyelination of L5 axons

To investigate the impact of demyelination at the level of single L5 axons, we recorded from visually identified L5 neurons in the primary somatosensory hindlimb region (S1HL) in slices from adult C57BL/ 6 mice, which were fed with $0.2 \%$ cuprizone for 5 weeks, a standard regime to obtain maximal oligodendrocyte death and loss of myelin protein expression (Kipp et al., 2009). Recorded cells were processed for biocytin and demyelination was assessed by immunofluorescence of MBP (essential for the formation of compact myelin; Boggs, 2006; Fig. 1A). Confocal $z$-projected images showed that although $0.2 \%$ cuprizone for 5 weeks reduced MBP expression prominently within the L5 and L6, patches of MBP could still be observed (Fig. 1A). Also when feeding mice for 9 weeks with $0.2 \%$ cuprizone, L6 was still immu- 
nopositive for MBP, indicating that $0.2 \%$ allows continuation of remyelination, consistent with previous work (Lindner et al., 2008). In contrast, $0.3 \%$ cuprizone feeding for 9 weeks induced a near-complete demyelination of the gray matter region (Fig. 1A).

To quantitatively examine the differences in myelin loss across the groups, we used confocal image analysis of MBP immunofluorescence. Analysis of the myelin content in L6 revealed that $0.3 \%$ cuprizone treatment for 9 weeks compared with control and $0.2 \%$ cuprizone for 5 weeks induced significantly more myelin loss (5.1 vs 50.4 and $13.9 \%$, respectively; Fig. $1 B, C$ ). Furthermore, myelination of single axons was quantitatively measured by calculating the percentage of axon length covered with MBP. Control L5 axons had an average onset of myelin at $45.7 \pm 2.1$ $\mu \mathrm{m}$ distance from the soma, at the end of the AIS, and myelination continued across the entire length examined (100\%, $n=10$; Fig. $1 D)$. In contrast, $0.2 \% / 5$ weeks cuprizone treatment caused a significant $\sim 70 \%$ loss of myelin and shifted the myelin onset to more distal locations in the axon $(276.6 \pm 40.6 \mu \mathrm{m}$ distance from the soma, $n=9$ axons, one-way ANOVA followed by Bonferroni's post hoc test, $p=0.0002)$. Additionally, nearly $\sim 35 \%(n=5$ of 14) of axons in $0.2 \% / 5$ weeks group were completely demyelinated. In comparison, within the $0.3 \% / 9$ weeks group, axons showed a $\sim 97 \%$ loss of myelin and $\sim 81 \%(n=13$ of 16$)$ of the axons labeled with MBP were completely demyelinated (Fig. $1 D$ ). In three MBP-positive axons $(n=3)$, the myelin onset occurred at significantly more distal locations from the soma $(0.3 \% / 9$ weeks, $448.3 \pm 101 \mu \mathrm{m}, n=3$ axons; compared with control, Bonferroni's post hoc test, $p<0.0001$; compared with $0.2 \% / 5$ weeks, $p=0.047)$. Despite the major loss of myelination, the axon morphology, as assessed by diameter, was not affected (axon diameter at $300 \mu \mathrm{m}$ from soma: control, $1.03 \pm 0.08 \mu \mathrm{m}$, $n=7 ; 0.2 \% / 5$ weeks, $0.76 \pm 0.04 \mu \mathrm{m}, n=4 ; 0.3 \% / 9$ weeks, $0.92 \pm 0.07 \mu \mathrm{m}, n=6$; one-way ANOVA, $p=0.09)$.

Together, the observed spatial and temporal dynamics of the $0.2 \% / 5$ weeks and $0.3 \% / 9$ weeks cuprizone treatment indicates that the two paradigms cause an "acute" or "chronic" loss of myelination in L5 axons, respectively.

\section{Morphological changes of L5 neurons in demyelination}

To examine whether demyelinated L5 pyramidal neurons of the somatosensory cortex exhibit dendritic morphological differences, neurons were labeled with biocytin for post hoc analysis. Morphological analysis showed that targeted neurons were always of the thick-tufted L5 pyramidal neuron type ( $n=38$ neurons; Fig. 2A). All analyzed neurons extended apical dendrites that terminated in dendritic tufts near the pia, with laminar position of the bifurcation points of primary apical dendrites typically observed near the borders of L2/3 and L4. Following chronic demyelination, L5 neurons had apical dendrites that were on average $\sim 100 \mu \mathrm{m}$ shorter compared with control and acute demyelination (Fig. $2 B$ ). The width of the apical dendritic tufts in neurons of cuprizone-treated animals was not different from that in control (Fig. 2B). Similarly, the diameter at the base of the apical dendrites of L5 neurons was not changed in cuprizonetreated animals (Fig. 2B).

We subsequently examined subdomains of the dendritic tree using Sholl plots. When comparing cuprizone-treated groups to control, it was found that chronic demyelination induced an increase in number of dendritic branches within a very small range spanning between 60 and $80 \mu \mathrm{m}$ from the center of the cell body (Fig. 2C).
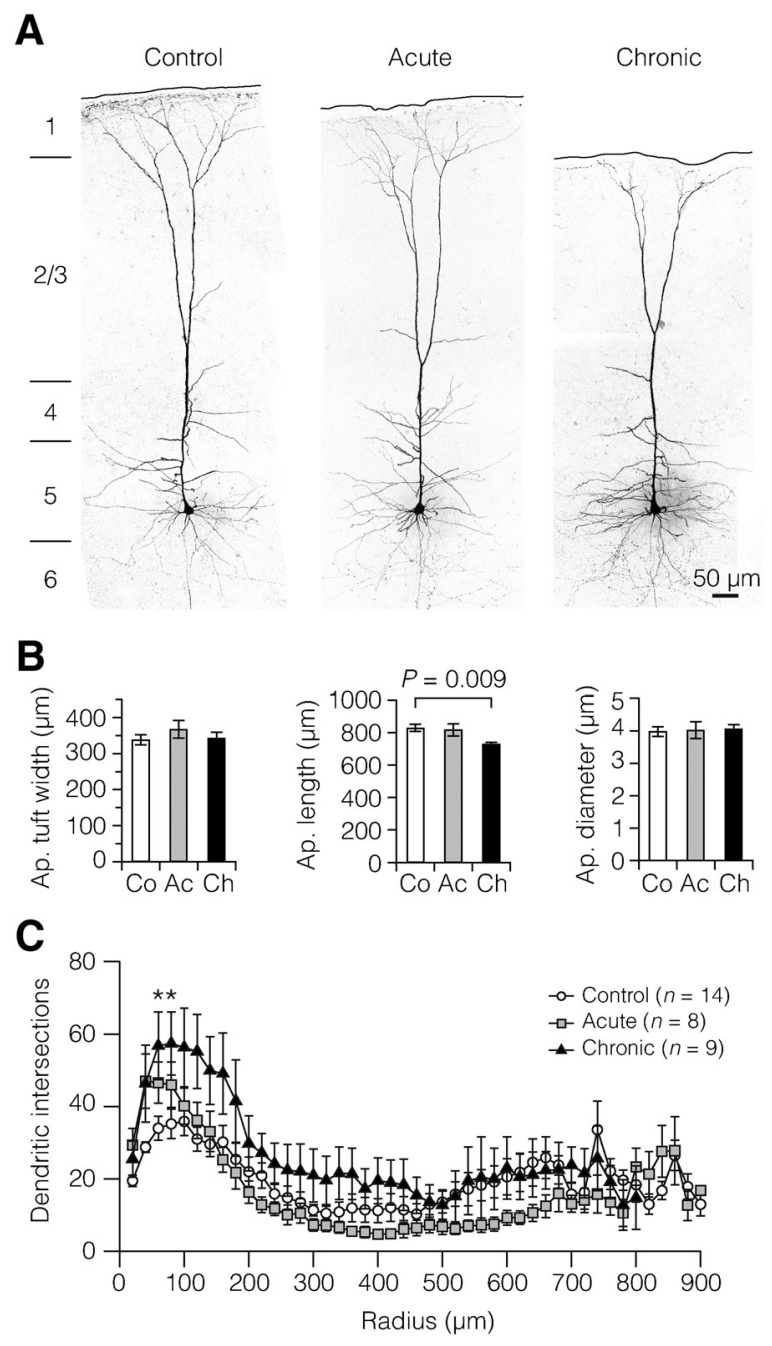

Figure 2. Chronic demyelination reduces the apical dendrites of $L 5$ neurons. $A$, Examples of z-projected confocal images of the morphology and whole-cell recorded thick-tufted L5 neurons in the somatosensory cortex. $\boldsymbol{B}$, Quantification of dendritic morphology showed that chronic demyelination reduces, probably as a result of cortical shrinkage, the apical dendritic length, as determined by the distance between tuft and soma. Apical tuft width: control (Co), $n=16$ neurons; acute (Ac), $n=10$; chronic (Ch), $n=12$. Apical length: $C 0, n=17 ; A c, n=$ 10; Ch, $n=12$. Apical diameter: $\mathrm{C}, n=17 ; \mathrm{Ac}, n=8 ; \mathrm{Ch}, n=12$. C, Sholl-plot analysis of dendritic segments within $20 \mu \mathrm{m}$ distance from the soma. Note the larger number of basal dendritic intersections in the chronic demyelinated group between 60 and $80 \mu \mathrm{m}$ from the soma. One-way ANOVA followed by Bonferroni's post hoc test, ${ }^{*} p=0.019(60 \mu \mathrm{m}),{ }^{*} p=$ $0.026(80 \mu \mathrm{m})$. Data are presented as mean \pm SEM.

Demyelination-induced ion channel redistribution in the AIS To examine whether loss of myelin affects the voltage-gated ion channel composition of the AIS, we performed immunofluorescence experiments on the slices in which L5 neurons were filled with Alexa Fluor during the whole-cell recordings $(n=97)$. Confocal images revealed an intense immunosignal for $\beta \mathrm{IV}$-spectrin, an anchoring protein together with ankyrin G stabilizing Nav1.6 channels in the AIS (Rasband, 2010), and showed that it was associated with the plasma membrane (Fig. $3 A$ ). Three-dimensional analysis of $z$ stacks showed that the average AIS onset of $\beta$ IVspectrin, relative to the edge of the soma, was $3.39 \pm 0.36 \mu \mathrm{m}$ in control cells (Fig. $3 A, B$ ). Acute and chronic demyelination led, however, to a significantly more proximal onset at $1.65 \pm 0.32$ $\mu \mathrm{m}$ and at $0.92 \pm 0.19 \mu \mathrm{m}$, respectively (Fig. $3 A, B$ ). The average length of $\beta I V$-spectrin expression, however, did not change (Fig. $3 C$ ). Next, we labeled brain slices with a Nav1.6 antibody (Fig. 
A
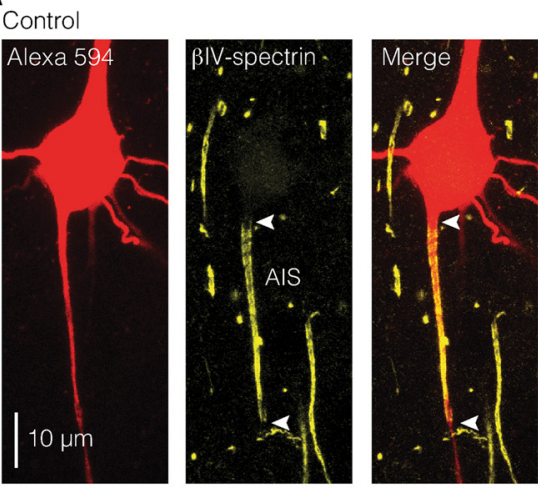

G
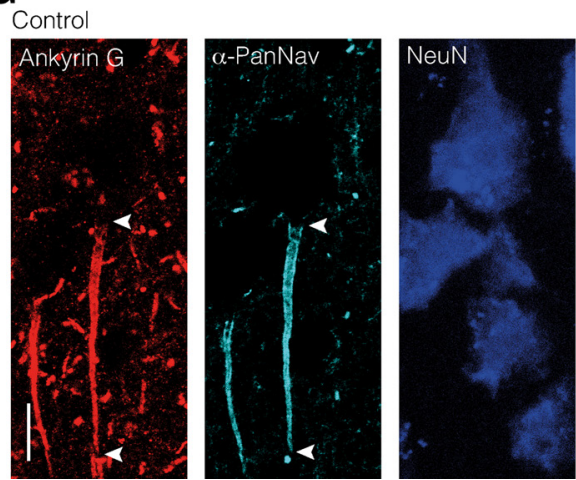

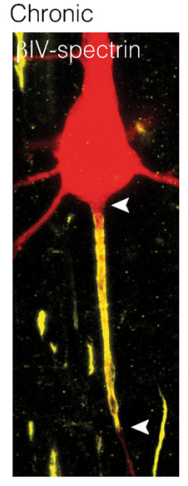

B

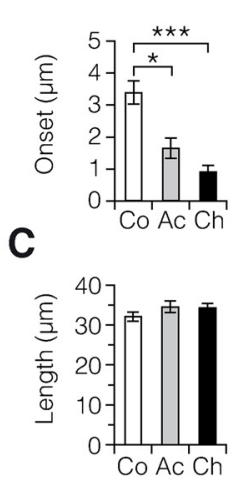

D
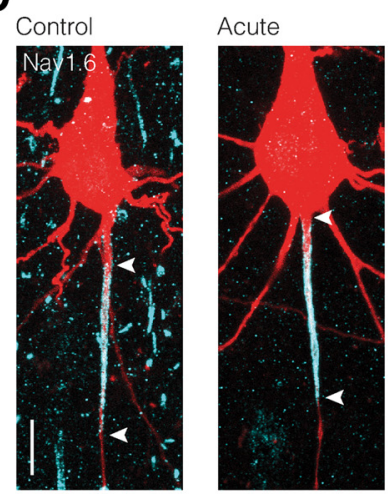

E

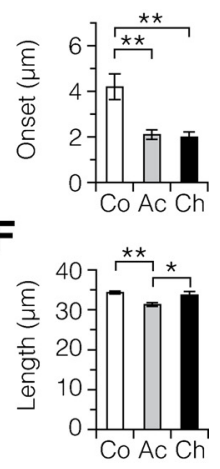

Figure 3. Demyelination-induced ion channel reorganization in the AIS. $A$, Single fluorescence channels and merged image of z-projected confocal scans of the soma and AIS morphology (Alexa Fluor 594 fill, red) and immunofluorescence labeling of $\beta$ IV-spectrin (yellow). White arrowheads indicate the onset and extent of $\beta$ IV-spectrin immunofluorescence identifying the AIS. $\boldsymbol{B}$, $\boldsymbol{C}$, Bar plots showing more proximal onset, but maintained length of expression of the AIS $\beta$ IV-spectrin immunofluorescence. Kruskal-Wallis followed by Dunn post hoc test. Onset: ${ }^{*} p=0.042,{ }^{* * *} p=$ 0.0003. Control, $n=8$; acute, $n=13$; chronic, $n=16 . \boldsymbol{D}-\boldsymbol{F}$, Nav1.6 immunofluorescence labeling (cyan) is significantly more proximal in its onset and changed in length in demyelinated axons. Onset: control versus acute, ${ }^{* *} p=0.0025$; control versus chronic, ${ }^{* *} p=0.0043$. Length: control versus acute, ${ }^{* *} p=0.0023 ;$ acute versus chronic, ${ }^{*} p=0.039$. Control, $n=14 ;$ acute, $n=23$; chronic, $n=17$. G-I, Confocal images of triple immunofluorescence of ankyrin $\mathrm{G}, \alpha$-PanNav, and NeuN. For both ankyrin $\mathrm{G}$ and $\alpha$-PanNav expression, length was maintained in acute demyelination. Mann-Whitney test. Ankyrin $\mathrm{G}, p=0.1647 ; \alpha$-PanNav, $p=0.099$. Control, $n=44$ AlSs; acute, $n=63$ AlSs.

$3 D)$. In control L5 neurons, the length of Nav1.6 immunosignal in the AIS was on average $34.3 \pm 0.4 \mu \mathrm{m}$ with a proximal onset of $4.2 \pm 0.6 \mu \mathrm{m}$ (Fig. $3 E, F$ ). In acutely demyelinated axons, the immunosignal length was significantly reduced and started at a more proximal site of onset (length, $31.3 \pm 0.5 \mu \mathrm{m}$; onset, $2.11 \pm$ $0.2 \mu \mathrm{m}$; Fig. $3 E, F)$. Chronically demyelinated L5 axons also showed a more proximal onset of Nav1.6 at $2.0 \pm 0.2 \mu \mathrm{m}$ but were not different in length of expression (Fig. $3 E, F$ ).

In addition to $\beta \mathrm{IV}$-spectrin and Nav1.6, we tripleimmunolabeled control and acute brain slices with ankyrin $G$, $\alpha$-PanNav, and NeuN (Fig. 3G). However, due to the required low-fixation regime, it was not possible to combine these two immunolabelings with neuron filling. Consistent with the $\beta I V-$ spectrin results, the average length of ankyrin G in acute demyelination was similar to that in control (control, $37.2 \pm 0.3 \mu \mathrm{m}$, acute, $37.9 \pm 0.4 \mu \mathrm{m}$, Fig. $3 H$ ). Interestingly, unlike the reduction of Nav1.6 expression in acute demyelination, we did not observe a reduction in $\alpha$-PanNav expression in the acute group (Fig. $3 I$ ). This could reflect an increase in expression of other Nav ion channels in the AIS, to compensate for the reduction observed in Nav1.6. However, immunolabeling of Nav1.2 ion channels did not show any specific fluorescent signal in the gray matter of both control $(n=18)$ and cuprizone-treated brain sections $(n=8$; data not shown).

Based on the $\beta \mathrm{IV}$-spectrin and Nav1.6 relocation, we hypothesized that the Kv7 (KCNQ)-gated ion channels, which are colocalized with Nav channels in both nodes of Ranvier and the AIS, and share a similar ankyrin G binding domain (Pan et al., 2006; Hill et al., 2008; Battefeld et al., 2014), may also be affected by myelin loss. In the control AIS, the Kv7.3 immunosignals relative to the soma edge had a distal onset of an average $12.8 \pm 1.2 \mu \mathrm{m}$ and signal length of $23.0 \pm 0.9 \mu \mathrm{m}$ (Fig. $4 A$ ). In contrast to Nav1.6, the AIS of acutely demyelinated axons displayed a similar Kv7.3 onset profile but channel expression significantly extended into the formerly myelinated internodes (length: acute, $35.9 \pm 4.2 \mu \mathrm{m}$; chronic, $35.8 \pm 4.1 \mu \mathrm{m}$; Fig. $4 B, C)$. Furthermore, when using triple fluorescence for Alexa 594, $\beta \mathrm{IV}$ spectrin, and Kv7.3, we found that in both acutely and chronically demyelinated axons the Kv7.3 immunosignal spread widely into the first internode without detectable levels of $\beta$ IV-spectrin (Fig. 4D).

\section{Demyelination-induced ion channel redistribution reduces AIS excitability}

The location of the AIS and its ion channel properties are major determinants of the AP voltage threshold and firing properties of neurons (Kole et al., 2008; Grubb and Burrone, 2010; Kuba et al., 2010; Battefeld et al., 2014). To examine the functional impact on neuronal excitability we explored the resting membrane parameters and AP properties of the thick-tufted L5 neurons in the three groups. The RMP in control L5 pyramidal neurons was on average $-67.9 \pm 0.26 \mathrm{mV}(n=100)$, but neurons from both the acute and chronic demyelinated group showed a $\sim 1-2 \mathrm{mV}$ more depolarized RMP (acute, $-66.93 \pm 0.31 \mathrm{mV}, n=93, p=0.025$; 
A
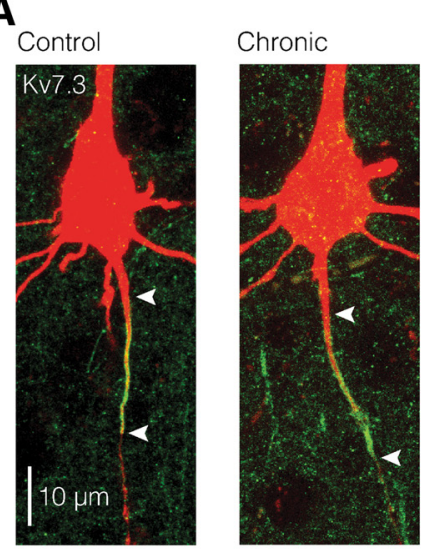

B

D
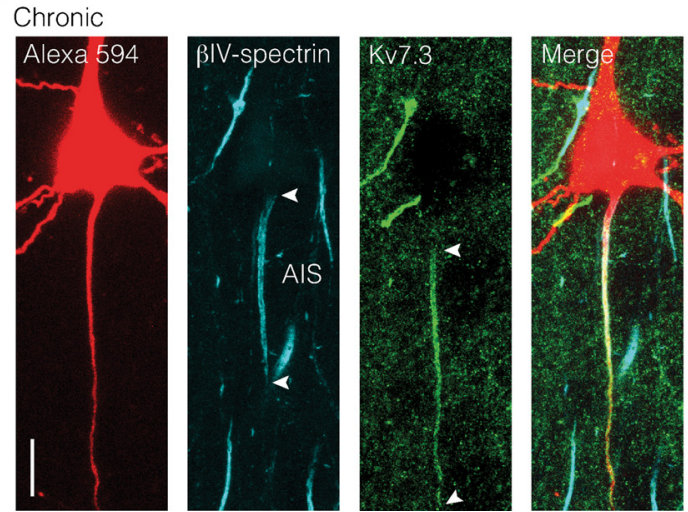

Figure 4. Kv7.3 ion channel expression continues into the first internode in demyelination. A-C, Kv7.3 immunofluorescence labeling (green) reveals a more extensive distribution of signal in demyelinated axons. Length: control versus acute, ${ }^{*} p=0.0375$; control versus chronic, ${ }^{*} p=$ 0.0396. Control, $n=8$; acute, $n=10$; chronic, $n=16$. Data are presented as mean \pm SEM. $\boldsymbol{D}$, Confocal $z$ projection of triple immunolabeling of a chronically demyelinated $L 5$ neuron, showing the diffuse expression of Kv7.3 into the formerly myelinated first internode independently of $\beta I V$-spectrin coexpression. This diffuse expression of Kv7.3 was found in both acute ( $n=3$ neurons) and chronic demyelinated ( $n=4$ neurons) group with expression length range of $44-82 \mu \mathrm{m}$.

chronic, $-66.22 \pm 0.46 \mathrm{mV}, n=54$; one-way ANOVA followed by Bonferroni's post hoc test, $p=0.0008)$. In the chronic demyelinated group, the input resistance was on average $49.5 \pm 1.4$ $\mathrm{M} \Omega(n=54$; Bonferroni's post hoc test, $p=0.024)$, significantly higher compared with control (control, $45.3 \pm 0.9 \mathrm{M} \Omega, n=101$; acute, $45.6 \pm 1.1 \mathrm{M} \Omega, n=90)$. This increase in input resistance was also accompanied by a lower rheobase of AP trains in chronic demyelinated neurons (control, $275.0 \pm 6.09, n=100$; acute, $263.2 \pm 9.12 n=87$; chronic, $243.1 \pm 8.5, n=51$; Bonferroni's post hoc test, $p=0.019$ ). These two changes are most likely associated with the reduced apical dendritic length in the chronically treated mice (Fig. 2).

The most consistent effect was that in both acute and chronic demyelinated L5 neurons the somatically recorded AP amplitude was $\sim 1-3 \mathrm{mV}$ smaller (control, $100.5 \pm 0.37 \mathrm{mV}, n=98$; acute, $99.1 \pm 0.47 \mathrm{mV}, n=77$, Bonferroni's post hoc test, $p=0.049$; chronic, $97.7 \pm 0.61 \mathrm{mV}, n=54$, Bonferroni's post hoc test, $p=$ 0.0001 ) without change in half-width (control, $0.60 \pm 0.007 \mathrm{~ms}$, $n=98$; acute, $0.60 \pm 0.007 \mathrm{~ms}, n=77$; chronic, $0.58 \pm 0.012 \mathrm{~ms}$, $n=53$; one-way ANOVA, $p=0.323$ ). The AP voltage threshold of demyelinated neurons showed a trend to depolarize following chronic cuprizone treatment (control, $-47.3 \pm 0.25, n=98$; acute, $-47.2 \pm 0.34, n=77$; chronic, $-46.3 \pm 0.40, n=53$; one-way ANOVA, $p=0.0682$ ). Closer examination of the AP rising phase showed that the initial component in the $\mathrm{d} V_{\mathrm{M}} / \mathrm{d} t$, which reflects the current flow from the AIS during onset of the somatic AP voltage waveform, was selectively reduced in chronically demyelinated neurons (control, $383.8 \pm 6.4 \mathrm{~V} \mathrm{~s}^{-1}, n=65$; chronic, $356.6 \pm 7.2 \mathrm{~V} \mathrm{~s}^{-1}, n=33$; Bonferroni's post hoc test, $p=$ $0.02)$.

To examine whether these properties were a result of cuprizone or the associated myelin loss, we bath applied $100 \mu \mathrm{M}$ cuprizone to control neurons in whole-cell current-clamp configuration in vitro and established the main properties. The RMP slightly hyperpolarized after $20 \mathrm{~min}$ of $100 \mu \mathrm{M}$ cuprizone bath application (control, $-67.9 \pm 1.3 \mathrm{mV}$; cuprizone, $-69.0 \pm 1.1 \mathrm{mV}, n=5$; paired Wilcoxon test, $p=0.04$ ). However, bath application of cuprizone had no significant effects on AP properties, including AP amplitude (control, $100.4 \pm 1.5 \mathrm{mV}$; cuprizone, $98.6 \pm 1.4 \mathrm{mV}, n=5$; paired Wilcoxon test, $p=0.17$ ).

Next, to test the hypothesis that the experimentally observed changes in AIS position or AIS length (Fig. 5) were associated with the group changes in AP generation, we took advantage of our correlated approach of recording APs from neurons that were also fluorescently labeled for $\beta I V$-spectrin or Nav1.6. We plotted the onset location of the AIS, varying between 0.5 and 8 $\mu \mathrm{m}$, against various parameters of excitability recorded from the same cell. The results showed that AIS onset significantly, and positively, correlated only with the AP amplitude in both control and demyelinated neurons (Fig. 5A,B). A linear fit of the pooled data from control and demyelinated neurons revealed a slope of

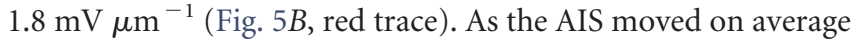
$\sim 2 \mu \mathrm{m}$, these results quantitatively predict a $3.6 \mathrm{mV}$ amplitude reduction, in accord with the $\sim 3 \mathrm{mV}$ AP amplitude reduction in demyelinated L5 neurons.

To test whether AIS length or location suffices to reduce the somatically recorded APs, we generated a computational model of a control L5 neuron, reconstructed at high resolution from confocal microscopy $z$ stacks (see Materials and Methods; Fig. $5 C)$. We took particular care to reconstruct the AIS at the precise onset distance $(3.9 \mu \mathrm{m})$ and length $(39 \mu \mathrm{m})$ based on the overlaid $\beta I V$-spectrin distribution. Next, to simulate the experimentally recorded AP, the peak Nav conductance densities in the AIS and soma were adjusted to reproduce the AP rising phase shape recorded from the specific cell (AIS: $9000 \mathrm{pS} \mu \mathrm{m}^{-2}$; soma: $500 \mathrm{pS}$ $\mu \mathrm{m}^{-2}$; Fig. 5D). Subsequently, both AIS onset location and length were varied between 0.1 and $12 \mu \mathrm{m}$ and between 29 and 39 $\mu \mathrm{m}$, respectively. The result of changing these model parameters revealed that both onset and length reduction depolarized the somatic AP voltage threshold and thereby reduced the AP amplitude. Changes in AIS length alone had a larger impact compared with changes in onset location (Fig. $5 E, F$ ). The impact of onset location was $0.17 \mathrm{mV}_{\mu \mathrm{m}}^{-1}$ on AP amplitude and $0.4 \mathrm{mV}^{-1} \mathrm{~m}^{-1}$ on AIS length. While the computationally predicted geometrical relationship is less steep compared with the experimentally ob-

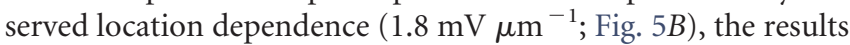
are in accord with the hypothesis that demyelination-induced geometrical changes reduce the capacity of the AIS Nav channels to generate inward current for the rising phase of the AP. Furthermore, the simulations predicted that the AP in the AIS at the distal end increased in half-width duration by $\sim 35 \%$ (control, $290 \mu \mathrm{s}$; demyelination, $390 \mu \mathrm{s}$ ) when the AIS has an onset directly from the cell body (Fig. $5 F$ ). 
Demyelination does not affect the AP initiation site in the AIS but reduces axonal conduction velocity

Based on the computationally predicted reduction in local AIS excitability, we next tested experimentally how local AP initiation in the AIS was affected by performing simultaneous patch-clamp recordings from the soma and axon. The axonal AP was assessed by recording the extracellular voltage (e-AP) at various distances from the soma along the axon during repetitive current injections (Fig. 6A; Palmer et al., 2010; Kole, 2011). The AP initiation site was identified by plotting the latency between the extracellular axonal AP onset and intracellular somatic AP, versus the distance of the axonal recording site (see Materials and Methods; Fig. 6B,C). In control neurons, the axonal initiation site of the AP was $\sim 27 \mu \mathrm{m}$ from the soma and not different from acutely demyelinated axons ( $\sim 25 \mu \mathrm{m}$; Fig. $6 D$ ). The e-AP latencies from locations distal from the AIS (>50 $\mu \mathrm{m})$ were fitted with a linear function yielding an estimate of axonal conduction velocity of $0.35 \mathrm{~m} \mathrm{~s}^{-1}$ in axons from cuprizone-fed mice, a $\sim 3$-fold velocity reduction compared with the control velocity $\left(1.1 \mathrm{~m} \mathrm{~s}^{-1}\right)$, consistent with the loss of myelin (Fig. 6E).

We next investigated the local properties of the e-AP waveform within the AIS (binned into $0,5,15,35 \mu \mathrm{m}$ ). Figure $6 F$ shows the e-AP waveforms, reflecting primarily the sum of the local $\mathrm{Na}^{+}$and $\mathrm{K}^{+}$ current densities, overlaid for distinct recording sites. Comparisons of e-AP waveforms from acute and chronic demyelinated neurons did not display any significant differences, and thus were pooled for further analysis. While the peak amplitudes of e-APs in demyelinated axons did not differ from the control e-AP amplitudes at any of the locations (0-35 $\mu \mathrm{m})$, the e-AP half-width was selectively increased at 5 and $15 \mu \mathrm{m}$ distance from the soma by $\sim 30$ and $\sim 40 \%$, respectively (Fig. $6 F, G$ ), consistent with the more proximal onset of Nav channel expression and impaired initiation of the local AIS AP (Fig. $5 F$ ).

Increased burst firing and spontaneous up-state-like events in demyelinated L5 neurons

If the AIS excitability is reduced, this may affect the input-output function of the demyelinated neurons. To test this we further analyzed the properties of subthreshold and suprathreshold excitability in both groups by current injections. The currentinjection-evoked excitability of L5 neurons can be broadly divided into intrinsic bursting (IB) and regular spiking neurons with nonadapting spike intervals of $\sim 100-200 \mathrm{~ms}$ (Hattox and Nelson, 2007; Kole, 2011). In control L5 neurons, steady current injections of just suprathreshold amplitudes either caused repet-
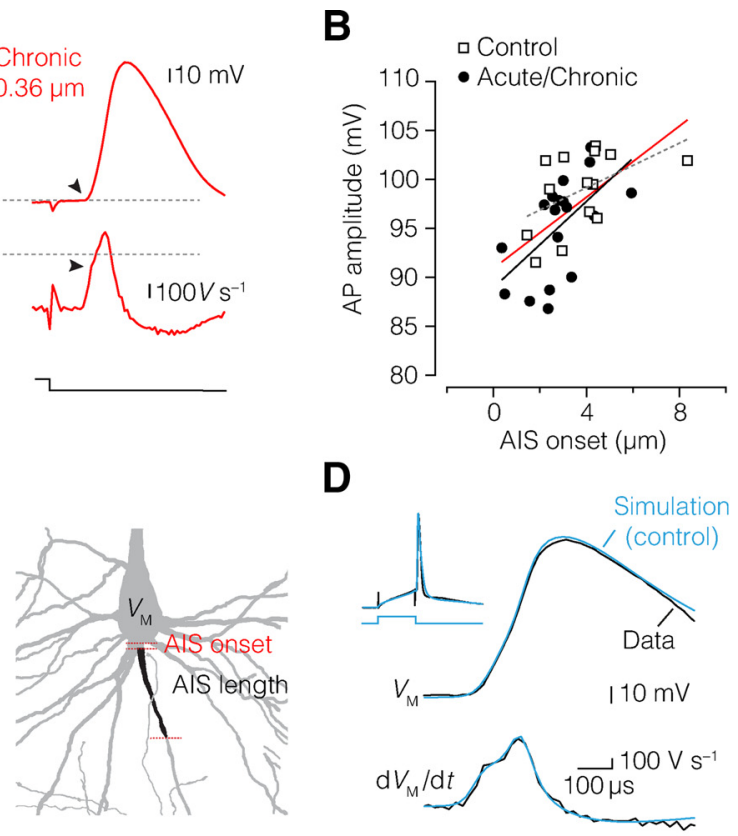

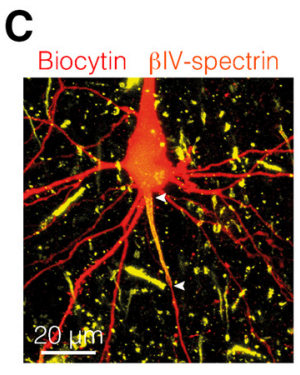

E

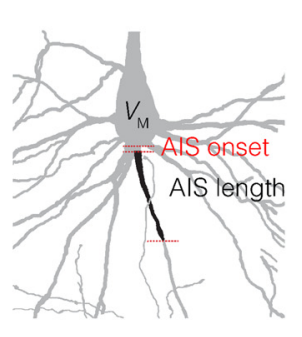

$\mathbf{F}$
Figure 5. AIS relocation and length reduction impairs AP initiation. $\boldsymbol{A}$, Neurons with proximal AIS onset generate APs with significantly lower amplitude. Top, Single AP from a control (black) and chronically demyelinated neuron (red) after a current injection (bottom), showing reduced AP amplitude in chronic demyelinated neuron with a more proximal AIS onset. Middle, Differentiated voltage, $\mathrm{d} V_{M} / \mathrm{d} t$, of the same traces, with a decreased initial peak (black arrowheads) reflecting a reduced current from the AIS during the onset of somatic voltage waveform. Control (black): onset, $4.4 \mu \mathrm{m} ; \mathrm{AP}$ amplitude, $103.1 \mathrm{mV} ; \mathrm{dV} / \mathrm{d} t$ $390.5 \mathrm{Vs} \mathrm{s}^{-1}$. Chronic (red): onset, $0.36 \mu \mathrm{m} ; \mathrm{AP}$ amplitude, $93.0 \mathrm{mV} ; \mathrm{dV} / \mathrm{d} /, 358.8 \mathrm{Vs}^{-1}$. B , AlS onset significantly correlated with AP amplitude in control (dotted gray line, closed circles; Spearman's $\rho=0.589, p=0.021, n=15$ ) and demyelinated neurons +90.9 (red line). Spearman's rank correlation $(\rho)$. C, Left, Confocal projection of the morphology of a thick-tufted L5 neuron labeled for biocytin and $\beta$ IV-spectrin. Right, 3D reconstruction of the same cell used for computational modeling. $D, 0$ verlaid and voltage waveforms from the experimental data (black) and simulated neuron (blue) for voltage (top) and time derivative (bottom). The inset shows the $6 \mathrm{~ms}, \sim 1 \mathrm{nA}$ current step, and voltage responses. Note the high degree of similarity between experiment and simulation. $\boldsymbol{E}$, Adjusting the AIS onset distance ( $x$-axis) and length of the AIS in the model (open and closed circles changes. $\boldsymbol{F}$, Examples of overlaid and aligned voltage traces of the simulated somatic AP waveform (top) and the AIS (bottom) corresponding to control parameters (blue) and reduced AIS onset and length values as shown in $\boldsymbol{E}$. Note the broader local AIS AP.

itive high-frequency bursts of 2-3 APs followed by regular spikes or a train of regular low-frequency APs only (Fig. $7 A$ ). IB of acutely demyelinated neurons was observed more frequently when compared with control and chronically demyelinated L5 neurons (Fig. $7 \mathrm{~B}, \mathrm{C}$ ). Interestingly, not only current-evoked excitability but also spontaneous activity was enhanced in demyelinated neurons. When monitoring the baseline activity of L5 neurons in current-clamp mode $(I=0)$ in epochs of 20-30 min, it was observed that demyelinated L5 neurons showed spontaneous activity, characterized by transient suprathreshold depolarizations triggering multiple APs or subthreshold depolarizing envelops, reminiscent of up-states reported for in vivo and in vitro recordings from L5 neurons (Fig. 7D; Sanchez-Vives and McCor- 
A

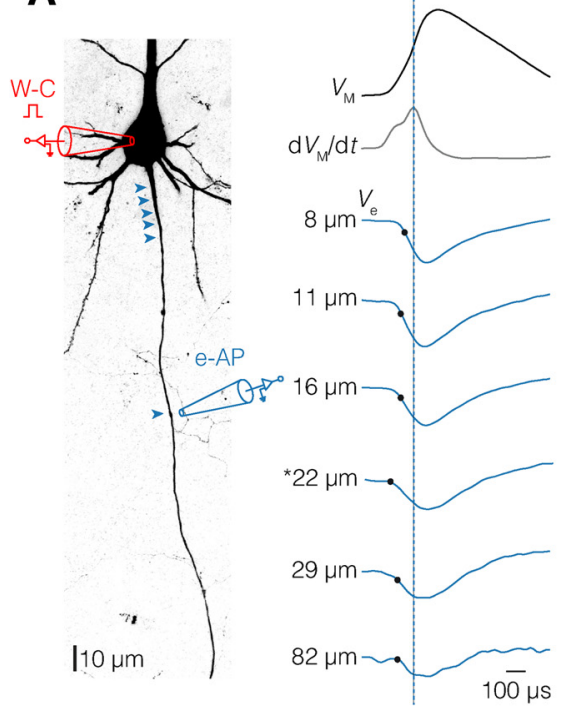

B

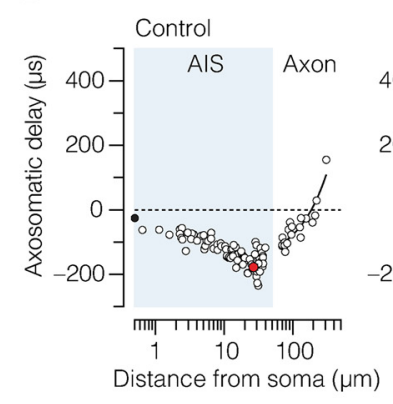

$\mathbf{F}$

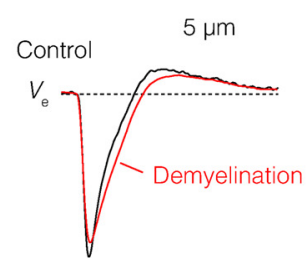

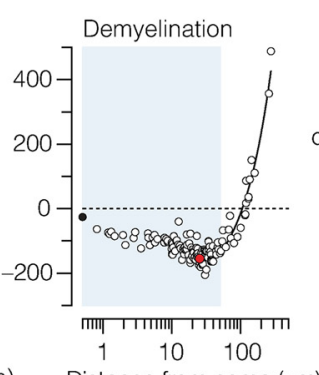

Distance from soma $(\mu \mathrm{m})$

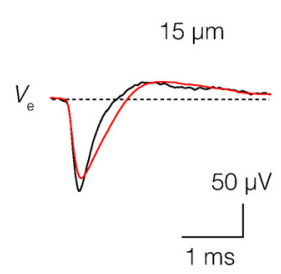

C

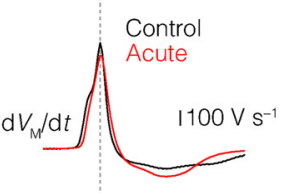

$v_{2} \int_{12 \mu v}$

$\overline{500 \mu s}$

G

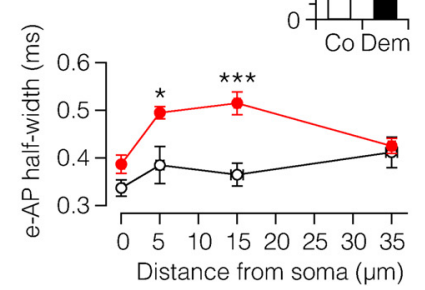

Figure 6. Maintained AIS initiation site, increased half-width, and reduced conduction velocity of axonal APs in demyelinated $L 5$ axons. $A, z$-projected confocal image of a $L 5$ pyramidal neuron illustrating the experimental design of whole-cell (W-C) configuration combined with e-AP recording. Locations of the recording sites for this example are indicated by blue arrowheads. e-AP traces (blue) from one typical recording aligned to the peak of the somatic $\mathrm{dV} V_{\mathrm{M}} / \mathrm{d} t$ (black). Black dots indicate the time when $20 \%$ of the local e-AP maximum is reached. Asterisk $(*)$ indicates the onset of AP. B, Pooled AP latency plotted versus axonal distance of the recording location in control (left, $n=30$ neurons) and demyelinated axons (right, $n=26$ acute and 2 chronic neurons). Red data point indicates the average initiation site. $C$, Top, Time derivative of APs from control (black) and acute demyelinated (red) $L 5$ neurons aligned at peak amplitude. Bottom, $\mathrm{e}-\mathrm{APs}$ recorded $\sim 160 \mu \mathrm{m}$ from the soma of respective neurons. Note the onset delay of the e-AP in the demyelinated axon due to reduction of conduction velocity as a consequence of myelin loss. Closed blue circles indicate the $20 \%$ onset of the local e-AP maxima. $D$, Location of AP initiation is maintained in demyelinated $L 5$ axons. Student's t test. Control, $26.6 \pm 1.5 \mu \mathrm{m}, n=10$; acute, $25.3 \pm 1.3 \mu \mathrm{m}, n=12 . E$, Bar plot showing the decreased conduction velocity (CV) in demyelinated neurons. Control, $1.1 \mathrm{~ms}^{-1}$; demyelination (acute and chronic), $0.35 \mathrm{~m} \mathrm{~s}^{-1}$. F, Average e-APs recorded at 5 and $15 \mu \mathrm{m}$ from the soma from control (black) and pooled demyelinated neurons (red, acute, and chronic). Note the broader e-AP half-widths of demyelinated neurons. $0 \mu \mathrm{m}$ : control, $n=11 ; n=14$ acute and 9 chronic. $5 \mu \mathrm{m}$ : control, $n=10 ; n=8$ acute and 9 chronic. $15 \mu \mathrm{m}$ : control, $n=10 ; n=10$ acute and 9 chronic. $35 \mu \mathrm{m}$ : control, $n=10 ; n=10$ acute and 9 chronic. G, Plot shows the average half-width of the $\mathrm{Na}^{+}{ }^{+}$component of the e-AP plotted against thelocation distance. Student'st test, ${ }^{*} p=0.02$, ${ }^{* * *} p=$ 0.0002. Co, Control; Ac, acute; Dem, demyelination (acute and chronic). Data are presented as mean \pm SEM.

A

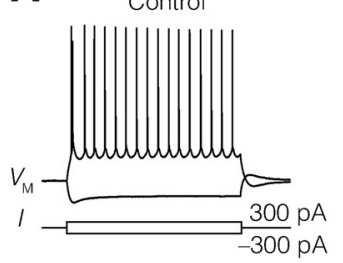

B

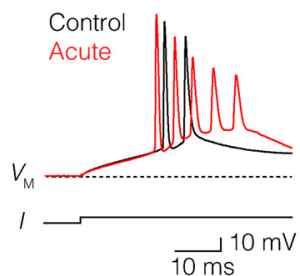

Acute

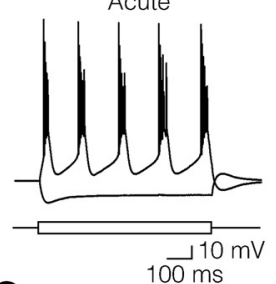

C

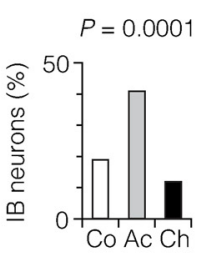

D

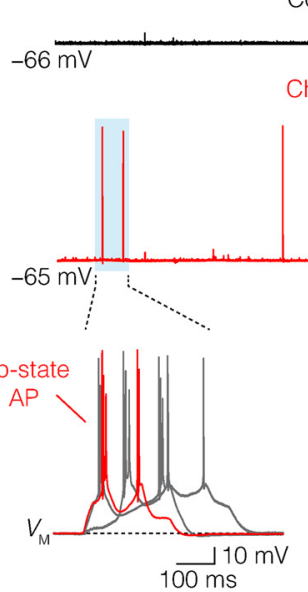

Control

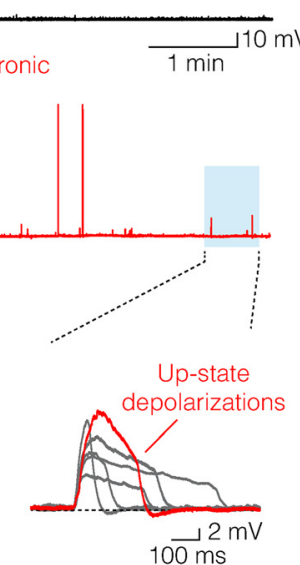

E

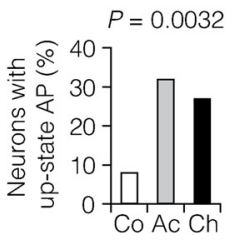

G

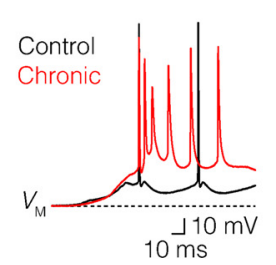

$\mathbf{F}$

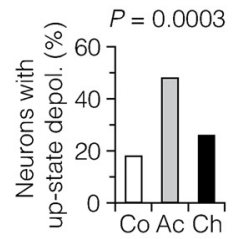

H

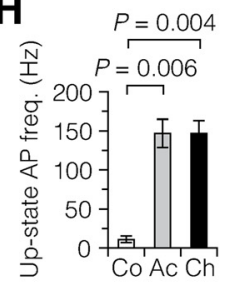

Figure 7. Demyelination increases burst firing and up-state-like depolarizations in L5 neurons. $A$, Suprathreshold current injection (bottom) induced burst firing in control (left) and acute demyelinated (right) neuron. $\boldsymbol{B}, 0$ verlaid voltage traces in $\boldsymbol{A}$ on an expanded time scale. Note the increase in number of APs in an acute demyelinated neuron (red) in response to the same stimulus, compared with control (black). C, Bar plot showing a significant increase in IB neurons in acute demyelination. $\chi^{2}$ Test. $\boldsymbol{D}$, Voltage traces of baseline activity at $\operatorname{RMP}\left(V_{\mathrm{M}}\right)$ from chronic demyelinated L5 neurons (middle, red), showing the occurrence of spontaneous up-state APs and depolarizations (blue area), compared with control neuron (top, black). Bottom, Overlaid voltage traces of up-state APs and depolarizations (middle, blue area). $\boldsymbol{E}$, Bar plot showing a significantly increased subpopulation of acute and chronic demyelinated L5 neurons generating up-state APs. $\chi^{2}$ Test. Control, $n=5$ of 61 ; acute, $n=25$ of 79 ; chronic, $n=27$ of 99 . $F$, Subpopulations of neurons with subthreshold up-state depolarizations increased in acute and chronic neurons. $\chi^{2}$ Test. Control, $n=11$ of 61 ; acute, $n=38$ of 79 ; chronic, $n=26$ of $99 . \mathbf{G}, \boldsymbol{H}$, Overlaid and aligned up-state AP voltage traces from control (black) and demyelinated neurons (red) on an expanded time scale. Note the high firing frequency of the demyelinated $(245.1 \mathrm{~Hz}$ ) neurons compared with control ( $23.4 \mathrm{~Hz})$. One-way ANOVA followed by Bonferroni's post hoc test. Control, $n=4$ neurons; acute, $n=19$ neurons; chronic, $n=24$ neurons. $C 0$, control; Ac, acute; Ch, chronic. Data are presented as mean \pm SEM.

mick, 2000; Beltramo et al., 2013). Up-state-like events are a characteristic feature of L5 neurons and were observed in a larger population of neurons from cuprizone-treated animals (up-state AP: control, $8 \%$; acute, $32 \%$; chronic, $27 \%$; up-state depolariza- tion: control, 18\%; acute, $48 \%$; chronic, $26 \%$; Fig. $7 E, F)$. These events also occurred more frequently within a single recording (up-state AP: control, $2.0 \pm 0.33 \mathrm{mHz}, n=5$; acute, $2.9 \pm 0.41$ $\mathrm{mHz}, n=25$; chronic, $4.8 \pm 0.75 \mathrm{mHz}, n=27$; one-way 
A

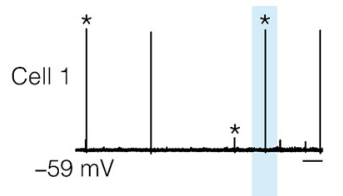

Ce $\|\left.\left. 2\right|_{-60 \mathrm{mV}} ^{4}|20 \mathrm{mV}|^{*}\right|^{*} \mathrm{~s}$

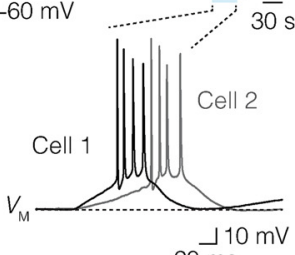

B

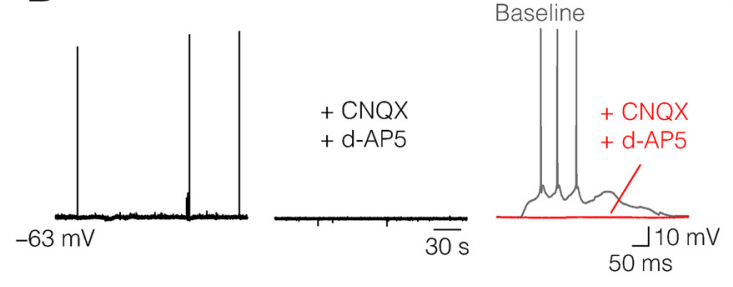

E
C

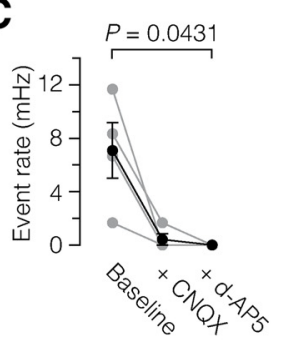

F

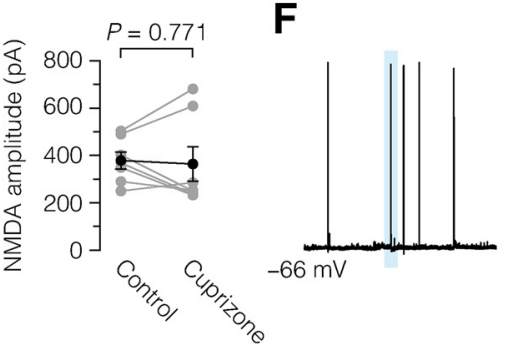

+20 nM TTX

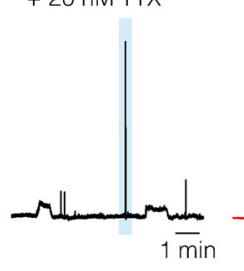

Baseline

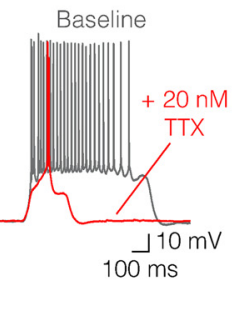

D
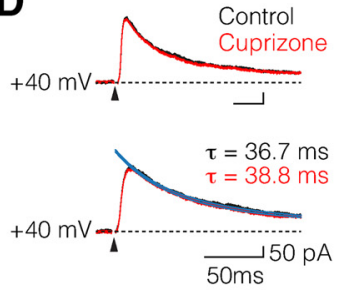

G

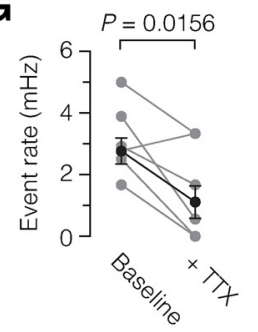

Figure 8. Glutamate-receptor and sodium channel dependence of demyelination-induced spontaneous up-state AP events. $\boldsymbol{A}$, Top, Simultaneous baseline current-clamp recording from two chronic demyelinated L5 pyramidal neurons (Cell 1 and Cell 2) shows correlated up-state AP events, indicative of a network-driven mechanism. Bottom, Temporally correlated up-state AP events (blue area) on an expanded time, showing the multiple APs of such events. Asterisk ( ${ }^{*}$ ) indicates synchronous events. $\boldsymbol{B}, \boldsymbol{C}$, Voltage traces of spontaneous up-state AP events in response to bath application of CNQX (20 $\mu \mathrm{m})$ and D-AP5 $(50 \mu \mathrm{m})$. Bath application of CNQX alone reduced the up-state AP event rate (baseline, $7.1 \pm 2.1 \mathrm{mHz}$; with CNQX, $0.42 \pm 0.42 \mathrm{mHz})$. Coapplication of CNQX and D-AP5 led to a complete block of the spontaneous events $(0.0 \mathrm{mHz}, n=4$ chronic neurons). Friedman followed by Dunn post hoc test. $\boldsymbol{D}, \boldsymbol{E}$, Top, Overlaid and aligned traces of basal NMDA currents, evoked by lateral stimulation in control condition (black) and during bath application of $100 \mu \mathrm{m}$ cuprizone (red, $n=7$ control neurons). Bottom, Same current traces on an expanded time scale fitted with double-exponential fit (blue lines). NMDA currents were recorded in the presence of $20 \mu \mathrm{m}$ CNQX and $2 \mu \mathrm{m}$ gabazine (SR 95531). Bath application of cuprizone did not affect the NMDA current amplitude or the decay time constant $(\tau)$. Black arrows indicate extracellular stimulus artifacts. $\boldsymbol{F}, \mathbf{G}, 20$ nM TTX significantly reduced the event rate of up-state AP events in demyelinated L5 neurons (baseline, $2.8 \pm 0.4 \mathrm{mHz}$; with TTX, $1.1 \pm 0.5 \mathrm{mHz}, n=2$ acute and 6 chronic neurons). Paired Wilcoxon test. Data are presented as mean \pm SEM.

ANOVA, $p=0.039$; up-state depolarization: control, $2.0 \pm 0.2$ $\mathrm{mHz}, n=11$; acute, $2.1 \pm 0.1 \mathrm{mHz}, n=38$; chronic, $2.7 \pm 0.3$ $\mathrm{mHz}, n=26$; one-way ANOVA, $p=0.043$ ) and the interspike frequency within the up-state AP was also significantly higher in demyelination $(\sim 100 \mathrm{~Hz}$; Fig. $7 G, H)$. Loss of myelination in the cuprizone model thus produces an increased excitability despite a decrease in AIS excitability.

\section{Glutamate receptor-dependent up-state-like depolarizations in demyelinated L5 neurons}

What underlies the increased excitability? We next aimed to examine whether up-state depolarizations after demyelination are dependent on network activity. Simultaneous recording from two horizontally adjacent demyelinated L5 neurons $(\sim 50 \mu \mathrm{m}$ apart, $n=3$ pairs) showed that up-state events could occur temporally correlated, consistent with synchronized network activity (Fig. 8A). To pharmacologically block synaptic activity, we bath applied glutamate receptor blockers and measured the impact on spontaneous AP generation. We first recorded a 10-20 min baseline activity in slices from cuprizone-treated mice and subsequently bath applied CNQX, a competitive AMPA/kainate receptor antagonist, which reduced the number of events (baseline, $\sim 7.1 \mathrm{mHz}$; CNQX, $\sim 0.42 \mathrm{mHz}$; Fig. 8 B, C). Subsequent addition of D-AP5, a selective NMDA receptor antagonist, led to a complete block (CNQX and D-AP5, $0 \mathrm{mHz}$; Fig. $8 \mathrm{~B}, \mathrm{C}$ ). Bath application of CNQX and D-AP5 also significantly reduced the number of upstate depolarizations in demyelinated neurons [baseline, $4.0 \pm$ $1.0 \mathrm{mHz}$; CNQX, $1.7 \pm 0.53 \mathrm{mHz}$; CNQX and D-AP5, $0.33 \pm 0.33$ $\mathrm{mHz}, n=5$ (acute); paired Friedman followed by Dunn post hoc test, $p=0.0089]$.

Based on these results, we hypothesized that synaptic glutamate receptor activity may be increased following demyelination. There is evidence that cuprizone, which is a copper chelator, may have also a direct impact on neurons by decreasing the free level of copper and thereby inducing persistent opening of the NMDA receptor (Stys et al., 2012). On the other hand, cuprizoneinduced demyelination for 6 weeks reduces AMPA receptor expression in the hippocampus, which may reduce the synaptic drive (Dutta et al., 2013). To test whether synaptic properties of L5 neurons were altered, we examined the interevent interval frequency and amplitudes of spontaneous postsynaptic potentials. The results showed, in contrast, that the postsynaptic potential frequency in demyelinated L5 neurons was not affected (control, $1.62 \pm 0.33 \mathrm{~Hz}, n=7$; demyelination, $1.54 \pm 0.15 \mathrm{~Hz}$, $n=6$ acute and 6 chronic; Student's $t$ test, $p=0.801)$. The postsynaptic potential amplitude in demyelinated neurons $(0.99 \pm$ $0.06 \mathrm{mV}, n=12$; Student's $t$ test, $p=0.177$ ) was also similar to that in control $(0.85 \pm 0.08 \mathrm{mV}, n=7)$.

To examine the role of direct cuprizone exposure on NMDA synaptic currents, we extracellularly stimulated intracortical fibers and recorded NMDA currents in the presence of $20 \mu \mathrm{M}$ CNQX and $2 \mu \mathrm{M}$ gabazine (SR 95531). In both control conditions and in the presence of $100 \mu \mathrm{M}$ cuprizone bath application, we did not observe any changes in amplitude (control, $379 \pm 36 \mathrm{pA}$; with cuprizone, $364 \pm 73 \mathrm{pA}, n=7$; paired Wilcoxon test, $p=0.813$; Fig. $8 D, E$ ) or decay time constant $(\tau$; control, $34.5 \pm 3.2 \mathrm{~ms}$; with cuprizone, $37.9 \pm 3.0 \mathrm{~ms}, n=7$; paired Wilcoxon test, $p=0.297$; Fig. $8 D$ ) of the NMDA currents. These results show that both long-term demyelination by cuprizone treatment and acute application of cuprizone do not change synaptic transmission of L5 pyramidal neurons.

Studies in the visual cortex showed that spontaneous pacemaker-like activity in the neocortex may be generated by the persistent $\mathrm{Na}^{+}$current (Le Bon-Jego and Yuste, 2007), and persistent $\mathrm{Na}^{+}$current has been implicated in axonal pathology in demyelination (Kapoor et al., 1997; Craner et al., 2004; Waxman, 2008). To test whether up-state APs were dependent on a subthreshold persistent $\mathrm{Na}^{+}$current in the demyelinated axon, we 
A
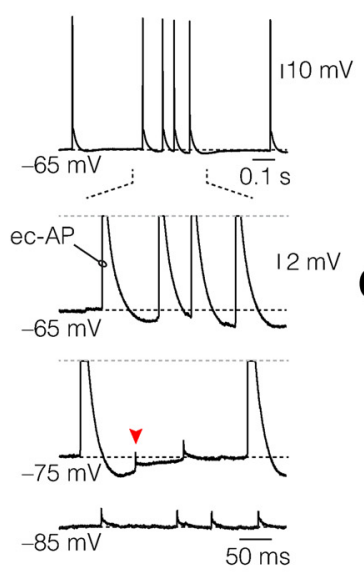

B

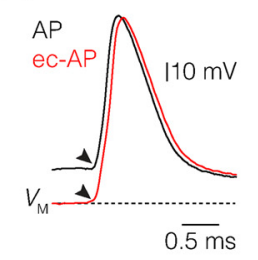

C

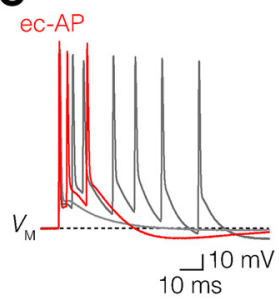

D
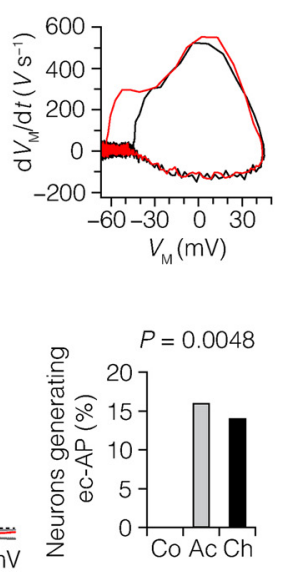
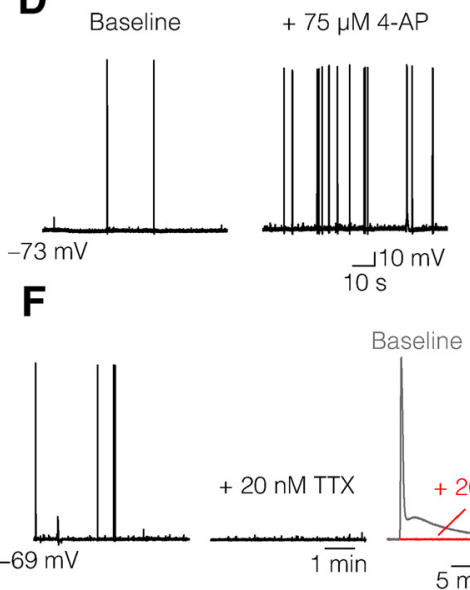

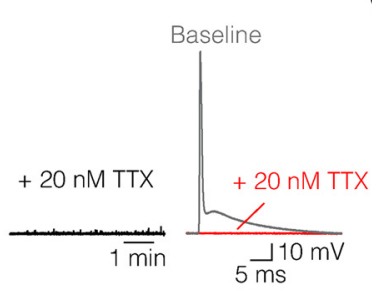

E

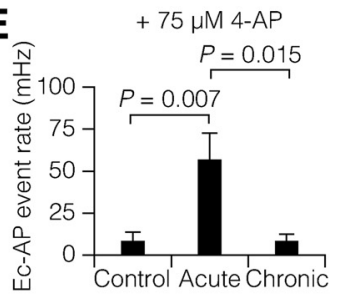

G

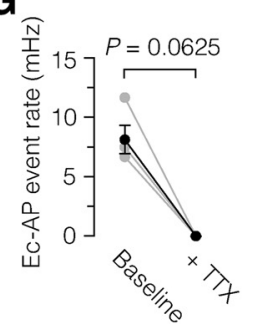

Figure 9. Ectopic AP generation in demyelinated $L 5$ axons. $\boldsymbol{A}$, Top, Spontaneous generation of ectopic AP (ec-AP) at resting potential. Bottom, Hyperpolarizing the membrane potential blocks invasion of the ec-APs into the soma and AIS, revealing an axonal spikelet (red arrowhead). $\boldsymbol{B}$, Left, Ec-AP waveform (red) has a distinctive abrupt voltage deflection from $V_{M}$ compared with the normal AP (black), which is preceded by a slow depolarizing ramp before reaching voltage threshold in the AIS (black arrowheads). Right, Phase plot of $d V_{M} / \mathrm{d} t$ versus $V_{M}$ of the same APs highlighting the longer interval between the AIS and somatic peaks of the ec-APs. C, Left, Three voltage traces of ectopic AP from demyelinated (acute and chronic) neurons overlaid and aligned at onset. Right, Bar plot of the percentage of ec-AP-generating neurons. $\chi^{2}$ Test, $p=0.0048$. Control, $n=0$ of $61 ;$ acute, $n=13$ of $79 ;$ chronic, $n=14$ of $99 . D, E$, Current-clamp baseline recording of 4 -AP-induced ec-AP generation from an acute demyelinated $\mathrm{L} 5$ neuron. Note the $\sim 7$-fold increase in ec-AP rate in acute demyelinated neurons (baseline, $4.2 \pm 3.5 \mathrm{mHz} ;$ with $4-\mathrm{AP}, 56.7 \pm 16 \mathrm{mHz}, n=8$ ) compared with control (baseline, $0 \mathrm{mHz}$; with 4-AP, $8.1 \pm 5.7 \mathrm{mHz}, n=10$ ) and chronic group (baseline, $0.3 \pm 0.3 \mathrm{mHz}$; with 4-AP, $8.2 \pm 4.4 \mathrm{mHz}, n=10$ ). Kruskal-Wallis test followed by Dunn post hoc test. $\boldsymbol{F}, \mathbf{G}$, Blocking of $\mathrm{Na}^{+}$persistent current with $20 \mathrm{~nm} \mathrm{TTX} \mathrm{prevented} \mathrm{ec-AP} \mathrm{generation} \mathrm{(baseline,} 8.13 \pm 1.2 \mathrm{mHz}$; with TTX, $0.0 \mathrm{mHz}$ ). Paired WilcoXon test. Acute, $n=2$; chronic, $n=$ 2. Data are presented as mean \pm SEM.

applied $20 \mathrm{~nm}$ of tetrodotoxin (TTX). TTX significantly reduced the rate of up-states but did not fully block them, suggesting that intrinsic conductances may contribute in part to the demyelination-induced increased up-state AP event rate (baseline, $2.8 \mathrm{mHz}$; plus $20 \mathrm{~nm}$ TTX, $1.1 \mathrm{mHz}$; Fig. 8 F, G). Bath application of $20 \mathrm{nM}$ TTX neither blocked nor modulated the event frequency of up-state depolarizations (baseline, $2.3 \pm 0.21 \mathrm{mHz}$; plus $20 \mathrm{~nm}$ TTX, $2.25 \pm 0.64 \mathrm{mHz}$; acute, $n=2$; chronic, $n=5$; paired Wilcoxon test, $p=0.8125)$. Thus, persistent $\mathrm{Na}^{+}$current may contribute to the amplification of the up-state depolarizations in demyelinated axons.

\section{Demyelination causes ectopic AP generation}

In the course of the long-duration recordings (Figs. 7, 8), it was furthermore observed that nearly $\sim 15 \%$ of the demyelinated L5 neurons exhibited ectopic APs (acute, 16\%; chronic, 14\%; Fig. $9 A-C)$. Consistent with the presumed ectopic origin, these APs had a nearly instantaneous voltage deflection from the RMP, reflecting axial current flow initiated distally in the axon and invading the AIS and somatodendritic region antidromically (Fig. 9B). Furthermore, when the soma was $10-25 \mathrm{mV}$ hyperpolarized, the AIS-somatodendritic component of the ectopic AP could be inactivated and isolated from the spontaneous distal axonal AP, appearing as a small $\sim 1 \mathrm{mV}$ spikelet at the soma (Fig. $9 A)$. Ectopic APs were never observed in control myelinated L5 neurons ( $0 \%$; 0 of 61 neurons; Fig. 9C). The average ectopic AP event rate during a recording was $\sim 7-10 \mathrm{mHz}$ in both acute $(7.1 \pm 1.7 \mathrm{mHz}, n=13)$ and chronic demyelination (10.1 \pm 3.6 $\mathrm{mHz}, n=14$; Mann-Whitney test, $p>0.999)$.

To further examine whether demyelinated L5 axons also have higher propensity of pharmacologically induced ectopic AP generation, we applied $75 \mu \mathrm{M}$ 4-aminopyridine (4-AP, a blocker of $\mathrm{Kv} 1$ and $\mathrm{Kv} 3$ voltage-gated $\mathrm{K}^{+}$channels) during current-clamp baseline recordings (Fig. 9D). Bath application of 4-AP induced ectopic APs in control neurons at rate of $\sim 8 \mathrm{mHz}$. However, in acutely demyelinated L5 axons, 4-AP induced nearly a $\sim 7$-fold increase in the rate of ectopic AP (Fig. 9E). In contrast, chronic demyelinated L5 axons displayed comparable sensitivity to 4-APinduced ectopic AP generation with control. Ectopic AP generation has previously been described in demyelinated axons from the sciatic nerve (Baker and Bostock, 1992; Felts et al., 1995) and has been thought to rely on activation of persistent $\mathrm{Na}^{+}$current at the site of demyelination (Kapoor et al., 1997). To test this, we bath applied $20 \mathrm{~nm}$ TTX, which depolarized the somatic AP voltage threshold by $17.2 \pm 1.2 \mathrm{mV}(n=2$ acute and 6 chronic) and reduced the AP amplitude by $45.7 \pm 6.4 \mathrm{mV}(n=8)$. At this concentration, TTX blocked the initiation of ectopic APs (baseline, 8.13; with TTX, $0.0 \mathrm{mHz}$; Fig. $9 F, G$ ), suggesting that activation of axonal $\mathrm{Na}^{+}$current is critically involved in the generation of demyelination associated ectopic APs.

Together, these results indicate that demyelination leads to decreased AP generation evoked from somatodendritic current inputs in the AIS while facilitating generation of $\mathrm{Na}^{+}$-dependent ectopic APs in axonal regions distally from the AIS.

\section{Demyelination-induced ion channel distribution in nodes of Ranvier}

The results above indicate that Nav channel-dependent distal axonal excitability is increased. While the majority of demyelinated L5 neurons generating ectopic APs had axons terminating in deep L6 or corpus callosum, four axons were cut between $\sim 150$ and $300 \mu \mathrm{m}$ distance from the soma $(237.5 \pm 28.9 \mu \mathrm{m})$, indicating that the source of ectopic APs in demyelinated L5 axons may be in the proximity of the first two nodes of Ranvier. To examine whether axonal hyperexcitability is caused by a reorganization of nodal ion channels, we investigated the expression of Nav1.6 at nodes of Ranvier in L5 axons, which were filled with Alexa 594 (Fig. 10A). Control nodes, identified by the position of branch points in the primary axon, were always positive for Nav1.6 (100\%, $n=17$ of 17 branch points, 6 axons; range: $74-$ 
A

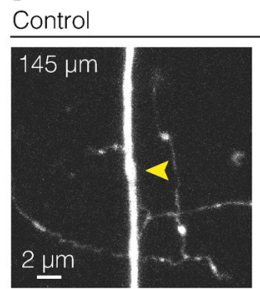

B

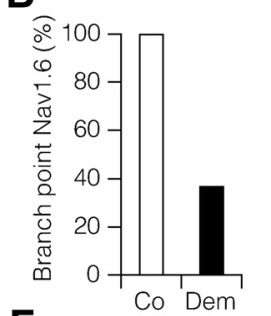

F

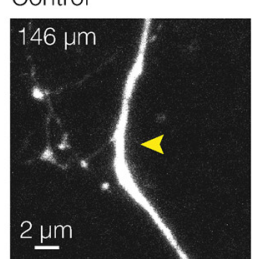

Alexa 594 Nav1.6

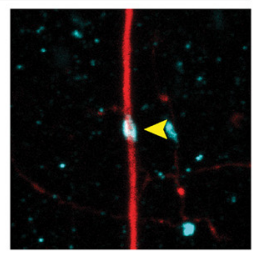

C
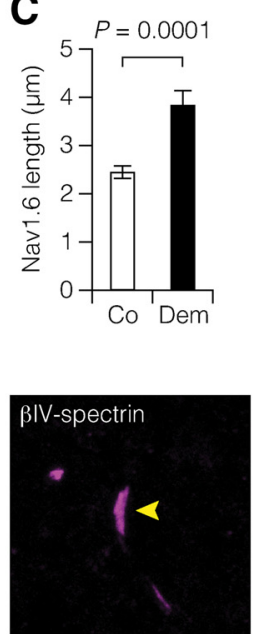

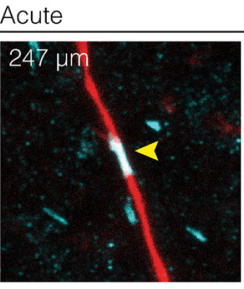

D
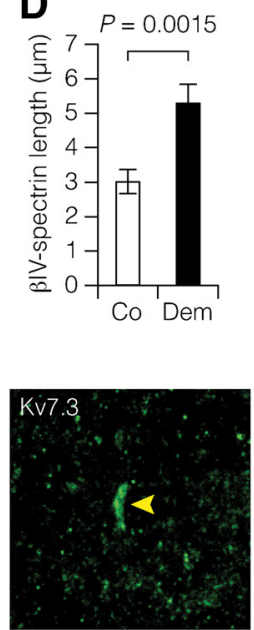

G

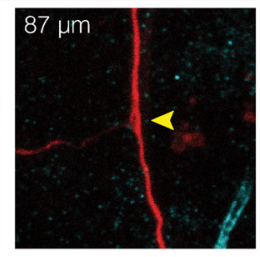

E
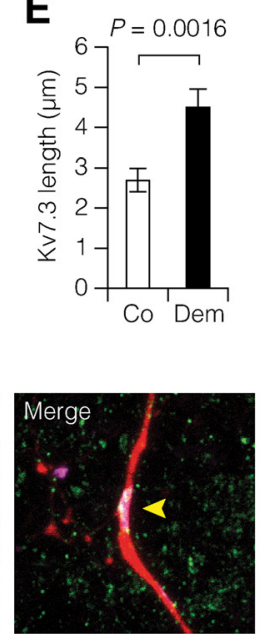

Chronic
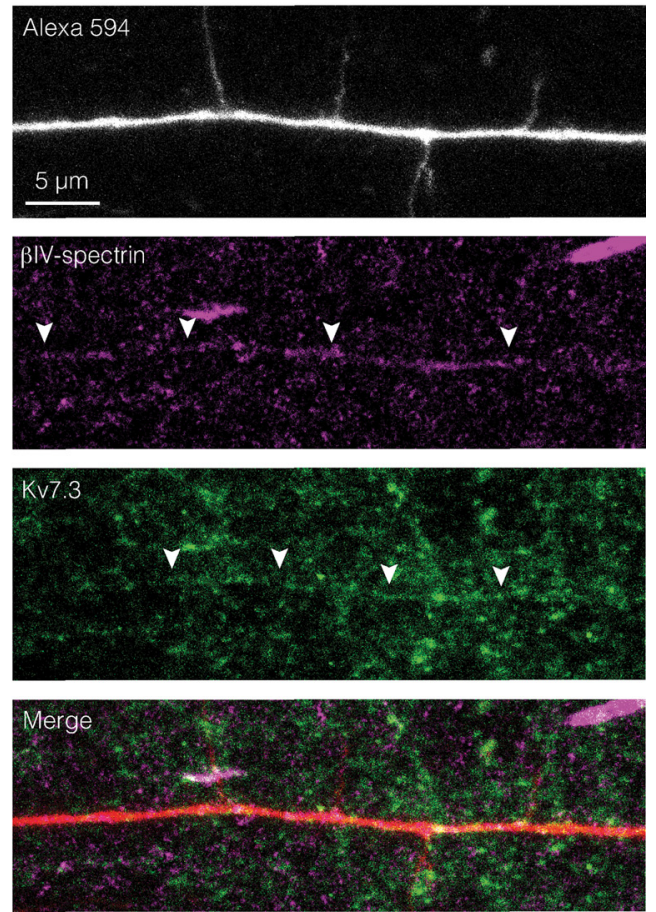

Figure 10. Heterogeneous redistribution of ion channels in demyelinated $L 5$ branch points and internodes. $A, z$-projected confocal images of double immunofluorescence labeling of a primary L5 axon (Alexa Fluor 594 fill) and Nav1.6 (cyan) at branch points in control and demyelinated axons. Yellow arrowheads indicate branch point location. $\boldsymbol{B}$, Bar graph showing the loss of Nav1.6-positive branch points of $L 5$ axons. Nav1.6-positive branch points: control, 17 of 17,6 axons; demyelination, $n=13$ of 35,18 axons; $\chi^{2}$ test, $p<0.0001$. C $E$, Bar plots of increased nodal expression length of Nav1.6, $\beta \mathrm{IV}$-spectrin, and Kv7.3 around nodes of demyelinated L5 axons. Nav1.6: control, $n=18$ nodes, 6 axons; demyelination, $n=24$ nodes, 12 axons. $\beta \mathrm{IV}$-spectrin: control, $n=15$ nodes, 5 axons; demyelination, $n=26$ nodes, 9 axons. Kv7.3: control, $n=19$ nodes, 6 axons; demyelination, $n=25$ nodes, 8 axons. Mann-Whitney test. Co, Control; Dem, demyelination (acute and chronic). Data are presented as mean \pm SEM. $\boldsymbol{F}, z$-projected confocal images of a branch point in control L5 axons filled with Alexa Fluor 594 and immunolabeled with $\beta I V$-spectrin (magenta) and Kv7.3 (green). G, Confocal z-projected images of a L5 axon double immunolabeled with $\beta \mathrm{IV}$-spectrin and Kv7.3. Note the loss of focal expression at axonal branch points but diffuse coexpression of $\beta \mathrm{IV}$-spectrin and Kv7.3 in the internodal regions (white arrowheads).

$392 \mu \mathrm{m}$ distance from the soma). In contrast, in demyelinated L5 axons nearly $63 \%$ of the nodes lacked Nav1.6 expression (Nav1.6-positive branch points: $n=13$ of 35, 18 axons; $\chi^{2}$ test, $p<0.0001$; Fig. 10B). Interestingly, 39\% $(n=7)$ of the axons contained both Nav1.6-positive and Nav1.6-negative branch points, indicating that the impact of demyelination leads to a large heterogeneity in nodal expression of ion channels. When Nav1.6 was present in distal axons, it was expressed over a significantly longer region compared with the more focal expression of control myelinated axons (Fig. 10C).

We also examined the expression of $\beta \mathrm{IV}$-spectrin and Kv7.3 (range: 87-327 $\mu \mathrm{m}$ distance from the soma; Fig. 10D-F). While $\beta \mathrm{IV}$-spectrin was detected in all control branch points, $\sim 48 \%$ of demyelinated branch points showed no $\beta \mathrm{IV}$-spectrin clustering ( $\beta$ IV-spectrin-positive branch points: control, $n=11$ of 11,4 axons; demyelination, $n=14$ of 27,9 axons; $\chi^{2}$ test, $p=0.0045$ ). In accord with $\beta \mathrm{IV}$-spectrin and Nav1.6 labeling, in demyelinated axons Kv7.3 was not present in $46 \%$ of the branch points (Kv7.3-positive branch points: control, $n=14$ of 14, 5 axons; demyelination, $n=14$ of 26, 8 axons; $\chi^{2}$ test, $\left.p=0.0024\right)$. In addition, $\beta \mathrm{IV}$-spectrin and Kv7.3 also displayed increased length of expression into the paranodal domains (Fig. 10D-F). Similar to Nav1.6 nodal heterogeneity, examination of $\beta \mathrm{IV}$-spectrin and Kv7.3 branch point expression revealed that a large percentage of axons contained both negative and positive branch points ( $\beta \mathrm{IV}$ spectrin, $n=6$ of 9 axons; Kv7.3, $n=5$ of 8 axons). Furthermore, in some demyelinated axons we observed that both $\beta \mathrm{IV}$-spectrin and Kv7.3 were also diffusely expressed along internodal regions but at the same time lost from branch points $(n=2$ axons; Fig. $10 G)$. In addition to the observation in fluorescently identified L5 axons, other nonfilled axons in L6 showed similar continuous $\beta \mathrm{IV}$-spectrin and Kv7.3 expression along internodal regions. However, brain sections of cuprizone-treated mice immunolabeled for ankyrin $\mathrm{G}(n=9), \operatorname{Nav1.6}(n=20), \operatorname{Nav1.2}(n=8)$, and $\alpha$-PanNav $(n=9)$ did not reveal continuous internodal expression (data not shown).

Together, these data show that Nav1.6, $\beta$ IV-spectrin, and Kv7.3 can both be lost and gained within nodal and paranodal regions but, in contrast to Nav1.6, the Kv7.3 channels redistribute extensively into the demyelinated internodes.

\section{Neurons with demyelinated axons are more susceptible to anomalous AP generation}

The observed divergence of nodal and internodal voltage-gated channel expression (Fig. 10) may lead to a highly heterogeneous excitability of the axon with longer diffuse expression of Nav1.6 channels underlying ectopic AP generation (Fig. 9). Demyelination exposes primary axons to the extracellular environment and possibly to local activity-driven $\mathrm{K}^{+}$and glutamate accumulation (Rasminsky, 1978; Kapoor et al., 1993). To investigate this possibility, we applied high concentrations of $\mathrm{K}^{+}$at fluorescently identified branch points and internodes $\left(10 \mathrm{~ms}\right.$ pulse, $\left[\mathrm{K}^{+}\right]=$ $140 \mathrm{~mm}$; Fig. 11A). The high- $\mathrm{K}^{+}$-evoked AP displayed ectopic characteristics with more hyperpolarized voltage threshold re- 
A

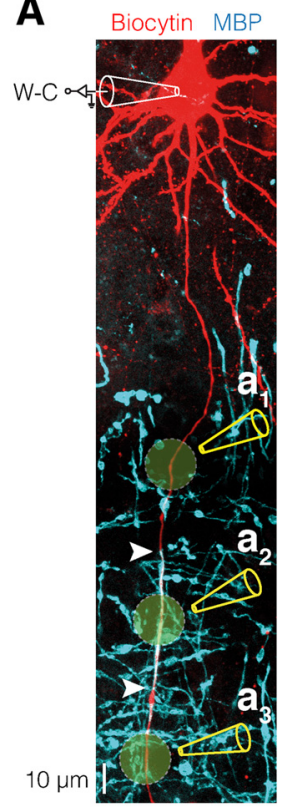

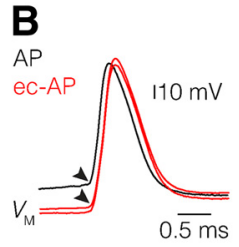
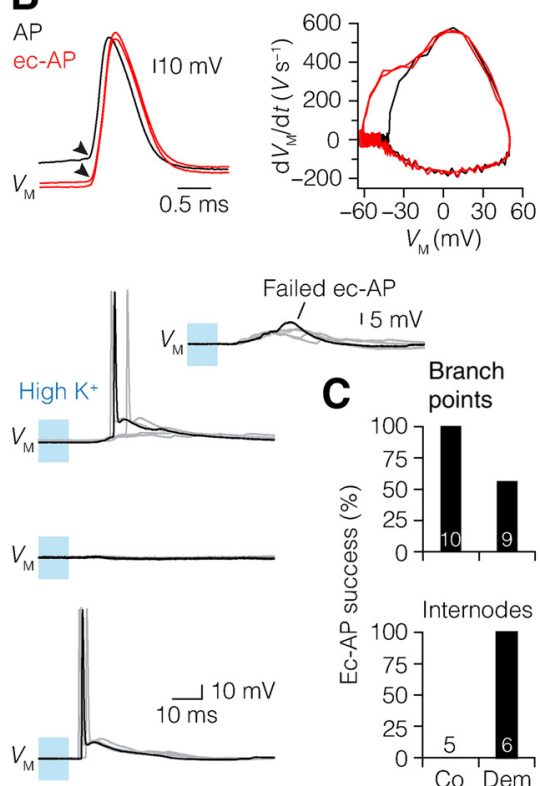

Ranvier and the AIS. To the best of our knowledge, these results provide the first evidence for a functional impact of myelin loss on the AIS and the occurrence of spontaneous intrinsic and network excitability of pyramidal neurons in the demyelinated neocortex.

\section{Loss of myelin causes highly heterogeneous changes in nodes of Ranvier}

By taking advantage of fluorescently identifying single gray matter L5 axons, allowing a longitudinal distribution profile analysis, and identifying nodal domains morphologically by their branch points, we found that Nav1.6 and Kv7.3 channels are highly heterogeneously affected. Even at neighboring branch points within one and the same demyelinated axon $\beta \mathrm{IV}$-spectrin, Nav1.6 and/or Kv7.3 could extend into the paranodes or disappear. The heterogeneity of the molecular properties of demyelinated nodes was consistent with the locally evoked nodal APs often failing at branch points. Surprisingly, in none of the L5 internodes did we find evidence for a Nav channel upregulation; that is, neither for $\alpha$-PanNav, nor Nav1.6, nor Nav1.2. Dispersion of the nodal Nav channel expression into paranodes and internodes is a hallmark of demyelinated axons, both in experimental models (Dupree et al., 2004; Crawford et al., 2009) and in the spinal cord and optic nerve of MS patients (Craner et al., 2004). While the immunosignals of Nav1.6 and $\alpha$-PanNav were always detected in the AIS, these primary antibodies do not show signals in the somatodendritic region, which is known to contain low densities of Nav channels. Therefore, the Nav antibodies used may not be able to detect expression of Nav channels at very low densities $(\leq 20$ channels $\left./ \mu \mathrm{m}^{2}\right)$ in demyelinated internodal regions. Even a modest Nav channel density increase in the internodes $(\sim 4 \%$ of the nodal density) can be sufficient to restore conduction of AP in demyelinated axonal regions (Shrager and Rubinstein, 1990). Additionally, it is also possible that upregulation of Nav channels in demyelinated internodes may be axon specific or occur at more chronic stages of demyelination.

In contrast to Nav channels, in the internodes of demyelinated L5 axons there was substantial evidence for diffuse Kv7.3 expression. A role of Kv7 channels in demyelinated axons has been computationally predicted and experimentally reported in axotomy-induced demyelination in peripheral axons (Coggan et al., 2010; Roza et al., 2011). Furthermore, on basis of in vivo axonal recordings in MS patients, the $I_{\mathrm{Ks}}\left(\right.$ or $\left.I_{\mathrm{M}}\right)$, mediated by Kv7.2/7.3 channels, is predicted to be upregulated ( $\mathrm{Ng}$ et al., 2008). The functional role of Kv7 in demyelinated axons remains to be further examined. Increased internodal $\mathrm{Kv} 7$, which is active at resting potential, may for example via a membrane potential hyperpolarization increase the availability of the transient Nav channels (Battefeld et al., 2014), ensuing continuation of axonal AP conduction.

\section{Compensatory ion channel expression in the AIS}

Two of our main findings were that despite the extensive myelin loss and dissolution of nodes of Ranvier, the AIS in the same axon always remained strongly immunopositive for Nav1.6 and Kv7.3, and AP generation from currents injected in the soma was only slightly impaired. The AIS structure changed, however, its location and reduced in length following acute, but not chronic, myelin loss. Recent studies showed that altered network activity, injury, or disease change the geometrical position of the AIS, leading to a homeostatic regulation of excitability (Kuba et al., 2006; Grubb and Burrone, 2010; Buffington and Rasband, 2011; Hinman et al., 2013). These results are consistent with a compen-

\section{Discussion}

The present study shows that cuprizone-induced myelin loss affects voltage-gated ion channel expression in the nodes of 
satory role of AIS relocation; in particular neurons with acutely demyelinated axons produced more intrinsic burst firing, had an increased sensitivity for generating ectopic APs, and were characterized by a more proximal AIS Nav channel onset and reduced length. A more proximal onset of the AIS will suffer from an increased current loss into the large somatodendritic domain acting as an axial current sink during rapid activation of AIS Nav channels, reducing the local AP rising phase in the AIS. The extent of AIS relocation in demyelination $(\sim 2 \mu \mathrm{m})$ was, however, insufficient to compensate for the increased intrinsic and network activity; both ectopic and spontaneously driven APs occurred more frequently in the L5 neurons in both experimental groups. Demyelination-induced AIS ion channel expression changes thus have a marginal impact on the overall neuronal excitability.

\section{Ectopic AP generation in demyelinated gray matter axons}

The present findings suggest that hyperexcitability is common in demyelinated gray matter axons. Ectopic APs have been previously reported during in vivo recordings within central and peripheral demyelinated axons (Rasminsky, 1978; Baker and Bostock, 1992; Felts et al., 1995; Kapoor et al., 1997) but never before in the cell bodies in the neocortex of demyelinating lesions. The physiological mechanisms underlying ectopic APs in demyelinated axons may include increased activation of persistent Nav channels, $\mathrm{K}^{+}$ion accumulation in the periaxonal/extracellular space, or an ephaptic coupling between the bare internodes (Rasminsky, 1978; Kapoor et al., 1993). Interestingly, while ectopic APs were observed in both cuprizone-treated groups, application of 4-AP induced a significantly larger increase of ectopic AP generation in acute demyelinated neurons, which were partially myelinated (Fig. 9E). These findings are consistent with the idea that ectopic APs are triggered during increased periaxonal $\mathrm{K}^{+}$accumulation in the vicinity of the MS lesion (Kapoor et al., 1993). In agreement with these mechanisms, a low concentration of TTX or local $\mathrm{K}^{+}$ application to demyelinated gray matter internodes was sufficient to eliminate and evoke ectopic APs, respectively.

While the occurrence of spontaneous ectopic APs in our neocortical slices was rare, on average $\sim 0.1 \mathrm{~min}^{-1}$, under in vivo conditions with increased activity, significantly higher local extracellular $\mathrm{K}^{+}$concentrations may accumulate and trigger ectopic APs and aberrant increased network activity. Furthermore, given the integrated role of astrocytes and oligodendrocytes in removing $\mathrm{K}^{+}$around nodal domains during axonal conduction (Rash, 2010), the loss of oligodendrocytes may lead to an impaired glial-mediated $\mathrm{K}^{+}$buffering in the demyelinated cortex. In addition to ectopic APs, the L5 neurons from cuprizonetreated mice also showed increased spontaneous excitability reminiscent of paroxysmal depolarizations reported at the soma from cortical pyramidal neurons in the connexin-32 knock-out mouse, which shows myelin defects (Sutor et al., 2000). The L5 axon provides both a recurrent excitatory feedback signal into the supragranular layers within the same column and in neighboring columns via the horizontally organized collaterals synchronizing activity and generating sensory-evoked network activity (SanchezVives and McCormick, 2000; Oberlaender et al., 2011; Beltramo et al., 2013). Spontaneous hyperexcitability, conduction failure, and/or ectopic APs in demyelinated axons may thus have a widespread impact on the intracortical computational functions of neocortical pyramidal neurons, the extent of which remains to be examined in vivo.

\section{Implications for therapeutic ion channel treatment in MS}

Cuprizone-induced lesions resemble some, but certainly not all, of the histopathological presentations of gray matter lesions in MS patients (Kipp et al., 2009; Clarner et al., 2012). The present evidence for axonal and neuronal hyperexcitability in the gray matter of cuprizone-treated mice is, however, noteworthy to relate to clinical observations. Demyelinating lesions and axonal injury in the gray matter are typically observed in 50\% of MS patients (Geurts et al., 2005; Geurts and Barkhof, 2008). MS patients often suffer from cognitive symptoms, including memory impairments, attention deficits, and slow sensory processing, which correlate with the extent of lesion load in the gray matter (Chiaravalloti and DeLuca, 2008; Fisniku et al., 2008). The finding of spontaneous APs generated outside of the AIS region may significantly debilitate the capacity to spatiotemporally encode sensory stimuli precisely. Identifying pharmacological means to prevent spontaneous ectopic generation of APs in gray matter lesions may thus benefit axonal function and/or injury and may alleviate some of the cognitive impairments.

Since clinical trials with sodium channel blockers failed to prevent neurodegeneration in MS (Waxman, 2008; Kapoor et al., 2010), the diffuse axonal Kv7.3 subunit channel expression along demyelinated gray matter internodes may provide interesting new directions for pharmacological intervention in demyelinating diseases. Kv7 channels are abundantly expressed in axons, are activated in the subthreshold voltage range, and strongly regulate AP frequencies. Such channels thus represent a promising alternative approach to modify demyelination-induced axonal excitability. Ezogabine (retigabine outside of the United States), a nonselective opener of Kv7.2-containing channels, has been approved for clinical use as an adjunctive treatment against partial epilepsy in adults (Stafstrom et al., 2011) and has been demonstrated to block ectopic APs in peripheral nerves and the neocortex (Straub et al., 2001; Roza and Lopez-Garcia, 2008). Conversely, XE-991 is clinically used as a cognitive enhancer and a highly selective Kv7.2/7.3 blocker (Wang et al., 2000). Examination of axonal excitability in the cuprizone model may thus provide a powerful experimental paradigm for exploration and development of rational therapies for gray matter axonal injury and the debilitating neurological and cognitive symptoms associated with demyelinating CNS disorders.

\section{References}

Baker M, Bostock H (1992) Ectopic activity in demyelinated spinal root axons of the rat. J Physiol 451:539-552. CrossRef Medline

Battefeld A, Tran BT, Gavrilis J, Cooper EC, Kole MH (2014) Heteromeric Kv7.2/7.3 channels differentially regulate action potential initiation and conduction in neocortical myelinated axons. J Neurosci 34:3719-3732. CrossRef Medline

Beltramo R, D’Urso G, Dal Maschio M, Farisello P, Bovetti S, Clovis Y, Lassi G, Tucci V, De Pietri Tonelli D, Fellin T (2013) Layer-specific excitatory circuits differentially control recurrent network dynamics in the neocortex. Nat Neurosci 16:227-234. CrossRef Medline

Boggs JM (2006) Myelin basic protein: a multifunctional protein. Cell Mol Life Sci 63:1945-1961. CrossRef Medline

Bostock H, Sears TA (1978) The internodal axon membrane: electrical excitability and continuous conduction in segmental demyelination. J Physiol 280:273-301. CrossRef Medline

Buffington SA, Rasband MN (2011) The axon initial segment in nervous system disease and injury. Eur J Neurosci 34:1609-1619. CrossRef Medline

Chiaravalloti ND, DeLuca J (2008) Cognitive impairment in multiple sclerosis. Lancet Neurol 7:1139-1151. CrossRef Medline

Clarner T, Diederichs F, Berger K, Denecke B, Gan L, van der Valk P, Beyer C, Amor S, Kipp M (2012) Myelin debris regulates inflammatory responses 
in an experimental demyelination animal model and multiple sclerosis lesions. Glia 60:1468-1480. CrossRef Medline

Coggan JS, Prescott SA, Bartol TM, Sejnowski TJ (2010) Imbalance of ionic conductances contributes to diverse symptoms of demyelination. Proc Natl Acad Sci U S A 107:20602-20609. CrossRef Medline

Craner MJ, Newcombe J, Black JA, Hartle C, Cuzner ML, Waxman SG (2004) Molecular changes in neurons in multiple sclerosis: altered axonal expression of Nav1.2 and Nav1.6 sodium channels and $\mathrm{Na}+/ \mathrm{Ca} 2+$ exchanger. Proc Natl Acad Sci U S A 101:8168-8173. CrossRef Medline

Crawford DK, Mangiardi M, Xia X, López-Valdés HE, Tiwari-Woodruff SK (2009) Functional recovery of callosal axons following demyelination: a critical window. Neuroscience 164:1407-1421. CrossRef Medline

Dupree JL, Mason JL, Marcus JR, Stull M, Levinson R, Matsushima GK, Popko B (2004) Oligodendrocytes assist in the maintenance of sodium channel clusters independent of the myelin sheath. Neuron Glia Biol 1:179-192. CrossRef Medline

Dutta R, Chomyk AM, Chang A, Ribaudo MV, Deckard SA, Doud MK, Edberg DD, Bai B, Li M, Baranzini SE, Fox RJ, Staugaitis SM, Macklin WB, Trapp BD (2013) Hippocampal demyelination and memory dysfunction are associated with increased levels of the neuronal microRNA miR-124 and reduced AMPA receptors. Ann Neurol 73:637-645. CrossRef Medline

Dzhashiashvili Y, Zhang Y, Galinska J, Lam I, Grumet M, Salzer JL (2007) Nodes of Ranvier and axon initial segments are ankyrin G-dependent domains that assemble by distinct mechanisms. J Cell Biol 177:857-870. CrossRef Medline

Felts PA, Kapoor R, Smith KJ (1995) A mechanism for ectopic firing in central demyelinated axons. Brain 118:1225-1231. CrossRef Medline

Felts PA, Baker TA, Smith KJ (1997) Conduction in segmentally demyelinated mammalian central axons. J Neurosci 17:7267-7277. Medline

Fisniku LK, Chard DT, Jackson JS, Anderson VM, Altmann DR, Miszkiel KA, Thompson AJ, Miller DH (2008) Gray matter atrophy is related to longterm disability in multiple sclerosis. Ann Neurol 64:247-254. CrossRef Medline

Foster RE, Whalen CC, Waxman SG (1980) Reorganization of the axon membrane in demyelinated peripheral nerve fibers: morphological evidence. Science 210:661-663. CrossRef Medline

Galiano MR, Jha S, Ho TS, Zhang C, Ogawa Y, Chang KJ, Stankewich MC, Mohler PJ, Rasband MN (2012) A distal axonal cytoskeleton forms an intra-axonal boundary that controls axon initial segment assembly. Cell 149:1125-1139. CrossRef Medline

Geurts JJ, Barkhof F (2008) Grey matter pathology in multiple sclerosis. Lancet Neurol 7:841-851. CrossRef Medline

Geurts JJ, Pouwels PJ, Uitdehaag BM, Polman CH, Barkhof F, Castelijns JA (2005) Intracortical lesions in multiple sclerosis: improved detection with 3D double inversion-recovery MR imaging. Radiology 236:254-260. CrossRef Medline

Grubb MS, Burrone J (2010) Activity-dependent relocation of the axon initial segment fine-tunes neuronal excitability. Nature 465:1070-1074. CrossRef Medline

Hallermann S, de Kock CP, Stuart GJ, Kole MH (2012) State and location dependence of action potential metabolic cost in cortical pyramidal neurons. Nat Neurosci 15:1007-1014. CrossRef Medline

Hattox AM, Nelson SB (2007) Layer V neurons in mouse cortex projecting to different targets have distinct physiological properties. J Neurophysiol 98:3330-3340. CrossRef Medline

Hill AS, Nishino A, Nakajo K, Zhang G, Fineman JR, Selzer ME, Okamura Y, Cooper EC (2008) Ion channel clustering at the axon initial segment and node of Ranvier evolved sequentially in early chordates. PLoS Genet 4:e1000317. CrossRef Medline

Hines ML, Carnevale NT (2001) NEURON: a tool for neuroscientists. Neuroscientist 7:123-135. CrossRef Medline

Hinman JD, Rasband MN, Carmichael ST (2013) Remodeling of the axon initial segment after focal cortical and white matter stroke. Stroke 44:182189. CrossRef Medline

Kapoor R, Smith KJ, Felts PA, Davies M (1993) Internodal potassium currents can generate ectopic impulses in mammalian myelinated axons. Brain Res 611:165-169. CrossRef Medline

Kapoor R, Li YG, Smith KJ (1997) Slow sodium-dependent potential oscillations contribute to ectopic firing in mammalian demyelinated axons. Brain 120:647-652. CrossRef Medline

Kapoor R, Furby J, Hayton T, Smith KJ, Altmann DR, Brenner R, Chataway J,
Hughes RA, Miller DH (2010) Lamotrigine for neuroprotection in secondary progressive multiple sclerosis: a randomised, double-blind, placebocontrolled, parallel-group trial. Lancet Neurol 9:681-688. CrossRef Medline

Kipp M, Clarner T, Dang J, Copray S, Beyer C (2009) The cuprizone animal model: new insights into an old story. Acta Neuropathol 118:723-736. CrossRef Medline

Kole MH (2011) First node of Ranvier facilitates high-frequency burst encoding. Neuron 71:671-682. CrossRef Medline

Kole MH, Stuart GJ (2012) Signal processing in the axon initial segment. Neuron 73:235-247. CrossRef Medline

Kole MH, Hallermann S, Stuart GJ (2006) Single $I_{\mathrm{h}}$ channels in pyramidal neuron dendrites: properties, distribution, and impact on action potential output. J Neurosci 26:1677-1687. CrossRef Medline

Kole MH, Ilschner SU, Kampa BM, Williams SR, Ruben PC, Stuart GJ (2008) Action potential generation requires a high sodium channel density in the axon initial segment. Nat Neurosci 11:178-186. CrossRef Medline

Kuba H, Ishii TM, Ohmori H (2006) Axonal site of spike initiation enhances auditory coincidence detection. Nature 444:1069-1072. CrossRef Medline

Kuba H, Oichi Y, Ohmori H (2010) Presynaptic activity regulates $\mathrm{Na}(+)$ channel distribution at the axon initial segment. Nature 465:1075-1078. CrossRef Medline

Le Bon-Jego M, Yuste R (2007) Persistently active, pacemaker-like neurons in neocortex. Front Neurosci 1:123-129. CrossRef Medline

Lindner M, Heine S, Haastert K, Garde N, Fokuhl J, Linsmeier F, Grothe C, Baumgärtner W, Stangel M (2008) Sequential myelin protein expression during remyelination reveals fast and efficient repair after central nervous system demyelination. Neuropathol Appl Neurobiol 34:105114. Medline

Mathis C, Denisenko-Nehrbass N, Girault JA, Borrelli E (2001) Essential role of oligodendrocytes in the formation and maintenance of central nervous system nodal regions. Development 128:4881-4890. Medline

Ng K, Howells J, Pollard JD, Burke D (2008) Up-regulation of slow K(+) channels in peripheral motor axons: a transcriptional channelopathy in multiple sclerosis. Brain 131:3062-3071. CrossRef Medline

Oberlaender M, Boudewijns ZS, Kleele T, Mansvelder HD, Sakmann B, de Kock CP (2011) Three-dimensional axon morphologies of individual layer 5 neurons indicate cell type-specific intracortical pathways for whisker motion and touch. Proc Natl Acad Sci U S A 108:4188-4193. CrossRef Medline

Ogawa Y, Rasband MN (2008) The functional organization and assembly of the axon initial segment. Curr Opin Neurobiol 18:307-313. CrossRef Medline

Palmer LM, Clark BA, Gründemann J, Roth A, Stuart GJ, Häusser M (2010) Initiation of simple and complex spikes in cerebellar Purkinje cells. J Physiol 588:1709-1717. CrossRef Medline

Pan Z, Kao T, Horvath Z, Lemos J, Sul JY, Cranstoun SD, Bennett V, Scherer SS, Cooper EC (2006) A common ankyrin-G-based mechanism retains KCNQ and NaV channels at electrically active domains of the axon. J Neurosci 26:2599-2613. CrossRef Medline

Rasband MN (2010) The axon initial segment and the maintenance of neuronal polarity. Nat Rev Neurosci 11:552-562. CrossRef Medline

Rash JE (2010) Molecular disruptions of the panglial syncytium block potassium siphoning and axonal saltatory conduction: pertinence to neuromyelitis optica and other demyelinating diseases of the central nervous system. Neuroscience 168:982-1008. CrossRef Medline

Rasminsky M (1978) Ectopic generation of impulses and cross-talk in spinal nerve roots of "dystrophic" mice. Ann Neurol 3:351-357. CrossRef Medline

Roza C, Lopez-Garcia JA (2008) Retigabine, the specific KCNQ channel opener, blocks ectopic discharges in axotomized sensory fibres. Pain 138: 537-545. CrossRef Medline

Roza C, Castillejo S, Lopez-García JA (2011) Accumulation of Kv7.2 channels in putative ectopic transduction zones of mice nerve-end neuromas. Mol Pain 7:58. CrossRef Medline

Sanchez-Vives MV, McCormick DA (2000) Cellular and network mechanisms of rhythmic recurrent activity in neocortex. Nat Neurosci 3:10271034. CrossRef Medline

Schmidt-Hieber C, Bischofberger J (2010) Fast sodium channel gating supports localized and efficient axonal action potential initiation. J Neurosci 30:10233-10242. CrossRef Medline

Shrager P, Rubinstein CT (1990) Optical measurement of conduction in single demyelinated axons. J Gen Physiol 95:867-889. CrossRef Medline 
Stafstrom CE, Grippon S, Kirkpatrick P (2011) Ezogabine (retigabine). Nat Rev Drug Discov 10:729-730. CrossRef Medline

Straub H, Köhling R, Höhling J, Rundfeldt C, Tuxhorn I, Ebner A, Wolf P, Pannek H, Speckmann E (2001) Effects of retigabine on rhythmic synchronous activity of human neocortical slices. Epilepsy Res 44:155-165. Medline

Stys PK, You H, Zamponi GW (2012) Copper-dependent regulation of NMDA receptors by cellular prion protein: implications for neurodegenerative disorders. J Physiol 590:1357-1368. CrossRef Medline

Susuki K, Chang KJ, Zollinger DR, Liu Y, Ogawa Y, Eshed-Eisenbach Y, Dours-Zimmermann MT, Oses-Prieto JA, Burlingame AL, Seidenbecher CI, Zimmermann DR, Oohashi T, Peles E, Rasband MN (2013) Three mechanisms assemble central nervous system nodes of Ranvier. Neuron 78:469-482. CrossRef Medline

Sutor B, Schmolke C, Teubner B, Schirmer C, Willecke K (2000) Myelination defects and neuronal hyperexcitability in the neocortex of connexin 32-deficient mice. Cereb Cortex 10:684-697. CrossRef Medline
Tian C, Wang K, Ke W, Guo H, Shu Y (2014) Molecular identity of axonal sodium channels in human cortical pyramidal cells. Front Cell Neurosci 8:297. CrossRef Medline

Tomassy GS, Berger DR, Chen HH, Kasthuri N, Hayworth KJ, Vercelli A, Seung HS, Lichtman JW, Arlotta P (2014) Distinct profiles of myelin distribution along single axons of pyramidal neurons in the neocortex. Science 344:319-324. CrossRef Medline

Wang HS, Brown BS, McKinnon D, Cohen IS (2000) Molecular basis for differential sensitivity of KCNQ and I(Ks) channels to the cognitive enhancer XE991. Mol Pharmacol 57:1218-1223. Medline

Waxman SG (2008) Mechanisms of disease: sodium channels and neuroprotection in multiple sclerosis-current status. Nat Clin Pract Neurol 4:159-169. CrossRef Medline

Waxman SG, Craner MJ, Black JA (2004) Na+ channel expression along axons in multiple sclerosis and its models. Trends Pharmacol Sci 25:584591. CrossRef Medline 